\title{
Onboard catalogue of known X-ray sources for SVOM/ECLAIRs ^
}

\author{
N. Dagoneau ${ }^{1}$, S. Schanne ${ }^{1}$, J. Rodriguez ${ }^{1}$, J.-L. Atteia ${ }^{2}$, and B. Cordier ${ }^{1}$ \\ ${ }^{1}$ Lab AIM - CEA, CNRS, Université Paris-Saclay, Université de Paris, 91191 Gif-sur-Yvette, France \\ e-mail: nicolas.dagoneau@cea.fr \\ 2 IRAP, Université de Toulouse, CNES, CNRS, UPS, Toulouse, France
}

Received 22 July 2020 / Accepted 19 October 2020

\begin{abstract}
The SVOM mission currently under development will carry various instruments, and in particular the coded-mask telescope ECLAIRs, with a large field of view of about 2 sr, operating in the $4-150 \mathrm{keV}$ energy band. The main goal of ECLAIRs is to detect highenergy transients such as gamma-ray bursts. Its onboard trigger software will search for new hard X-ray sources appearing in the sky, as well as peculiar behaviour (e.g. strong outbursts) from known sources, in order to repoint the satellite to perform follow-up observations with its onboard narrow-field-of-view instruments. The presence of known X-ray sources must be disentangled from the appearance of new sources. This is done with the help of an onboard source catalogue, which we present in this paper. As an input we use catalogues of X-ray sources detected by Swift/BAT and MAXI/GSC and we study the influence of the sources on ECLAIRs' background level and on the quality of the sky-image reconstruction process. We show that the influence of the sources depends on the pointing direction on the sky, on the energy band, and on the exposure time. In the Galactic centre, the contribution from known sources largely dominates the cosmic X-ray background, which is, on the contrary, the main background in sky regions lacking strong sources. We also demonstrate the need to clean the contributions of these sources in order to maintain a low noise level in the sky images and to maintain a low threshold for the detection of new sources without introducing false triggers. We briefly describe one of our cleaning methods and its challenges. Finally, we present the overall structure of the onboard catalogue and the way it will be used to perform the source cleaning and disentangle detections of new sources from outbursts of known sources.
\end{abstract}

Key words. instrumentation: miscellaneous - telescopes - catalogs - X-rays: general - techniques: image processing

\section{Introduction}

The most violent and energetic phenomena of the Universe usually emit copious amounts of high-energy radiation (typically $\mathrm{X}$ - and gamma-ray photons). These events are also transient and unpredictable, and some can occur on very short (sub-second) timescales. Dedicated instruments and specific observational strategies are therefore needed to capture those events. SVOM (Space-based multi-band astronomical Variable Objects Monitor, Wei et al. 2016) is a French-Chinese mission dedicated to the detection and follow-up of short flashes of hard $\mathrm{X}$-ray and gamma-ray photons called gamma-ray bursts (GRBs) and of other high-energy transients. SVOM is currently under development and planned to be operational after mid-2022.

The SVOM project involves both space-based and groundbased telescopes. Figure 1 shows the SVOM space platform which includes four space instruments: the ECLAIRs telescope, the Gamma Ray-burst Monitor (GRM), the Microchannel X-ray Telescope (MXT), and the Visible Telescope (VT). The SVOM mission also includes ground-based telescopes: a set of Ground Wide-Angle Cameras (GWACs) and two dedicated Ground Follow-up Telescopes (GFTs). These are reported schematically in Fig. 1 along with the main contributing countries.

The goal of the SVOM core program is the multi-wavelength study of GRBs, including the prompt and the afterglow emission, in order to obtain a complete sample of well-characterised

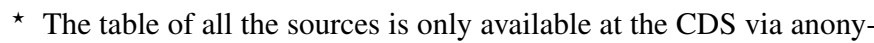
mous ftp to cdsarc.u-strasbg.fr $(130.79 .128 .5)$ or via http: //cdsarc.u-strasbg.fr/viz-bin/cat/J/A+A/645/A18
GRBs including redshift measurements for about two-thirds of the total sample. First, the GRB prompt emission is detected; its gamma-ray light curve and spectrum are characterised with the wide-field instruments ECLAIRs and GRM onboard, and these are simultaneously studied in the visible band with the GWAC on the ground, which, whenever possible, points at the same sky region as the onboard wide-field instruments. After the onboard GRB detection and a first automatic estimate of its best celestial coordinates by ECLAIRs, the spacecraft will slew to the GRB position, and the afterglow will be observed by the narrow-field instruments, namely MXT in the X-rays and VT in the visible band. Simultaneously, the GRB alert including its position will be transmitted to the ground through a VHF antenna, and the GFTs will perform follow-up observations in the visible and near-infrared bands. The GRB alerts and their subsequent refined positions obtained by the SVOM follow-up telescopes are quickly and publicly distributed to the whole community of interested observers of the transient sky. This strategy will enhance the number of accurately localised bursts, which is essential for fruitful follow-up observations with large groundbased facilities, including spectrometers on large ground-based telescopes, allowing for the redshift of those events to be determined.

In addition to the GRB detection and observation as part of its core program, SVOM will also carry out pre-planned observations in its so-called general program. This program includes known-source observations with the narrow-field-of-view instruments (MXT, VT, and GFTs) such as X-ray binaries (XRBs), AGNs, blazars, ultra-luminous X-ray sources, and cataclysmic variables, as well as wide-field surveys with ECLAIRs and 


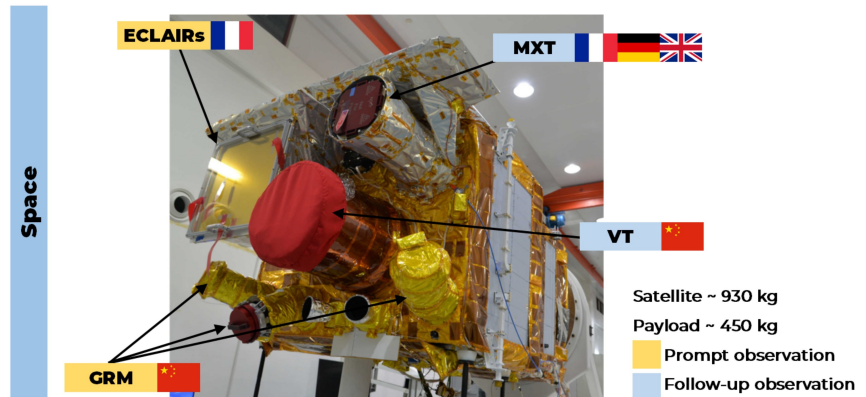

F-GFT

C-GFT GWAC *:

Fig. 1. Overview of the SVOM platform. The wide-field-of-view instruments are shown in orange boxes. The narrow-field-of-view instruments are depicted in blue boxes. The photo shows the structural and thermal models of the instruments on the qualification model of the satellite (CNES/SECM).

GRM. These observations will address some questions about the physics behind the emission processes in these objects (accretion through disks, ejection in jets). Also, following detection by other instruments, SVOM will perform fast observations of targets of opportunity for transient follow-up or optical counterpart searches of multi-messenger events. Further details of the SVOM prospects on GRB science, rapid follow-up observations, and observatory science are provided in Wei et al. (2016).

The SVOM spacecraft will be placed in a quasi-circular low-earth orbit (altitude $\approx 625 \mathrm{~km}$ ) with an inclination of about $30^{\circ}$. Its attitude (orientation with respect to an inertial reference frame) was optimised to follow the so-called "B1 law" which ensures an anti-solar pointing in order to protect the payload from the Sun light and guarantees that the field of view is simultaneously observable by ground instruments in the night hemisphere of the Earth. This law also avoids the presence of the Galactic plane (Galactic latitudes $|b|<10^{\circ}$ ) and the very bright X-ray source Scorpius X-1 (Sco X-1 in the following) in ECLAIRs' field of view, with a margin of $1^{\circ}$. As a consequence of this orbit and pointing strategy, the Earth will regularly cross the field of view of the instruments and influence their background level and shape. In our study, neither the Sun avoidance constraint nor the margin around Sco X-1 is considered and the following figures and values may differ slightly from those given in other communications. In the nominal phase of the mission (the first three years), the observing time outside the considered B1 law is $60 \%$ of the useful mission time and increases to more than $74 \%$ in the extended phase.

Figure 2 represents the expected exposure maps for the nominal (first three years) and extended phases of the mission, while Fig. 3 is the translation in terms of individual pointings (attitude of the instruments optical axis). Even if the Galactic poles have a much larger exposure time during the nominal phase than the Galactic centre (Fig. 2 top), this dichotomy is smoothed during the extended phase (Fig. 2 bottom), and the number of pointings outside the B1 law is significantly increased, particularly towards the Galactic centre and plane (Fig. 3).

The ECLAIRs space telescope, mainly dedicated to GRB detection and localisation, is a coded-mask aperture telescope operating in the energy range from 4 to $150 \mathrm{keV}$ (Godet et al. 2014). With such a low energy threshold, it is particularly well suited for the detection of X-ray-rich GRBs and highly redshifted GRBs. Figure 4 shows a schematic representation of
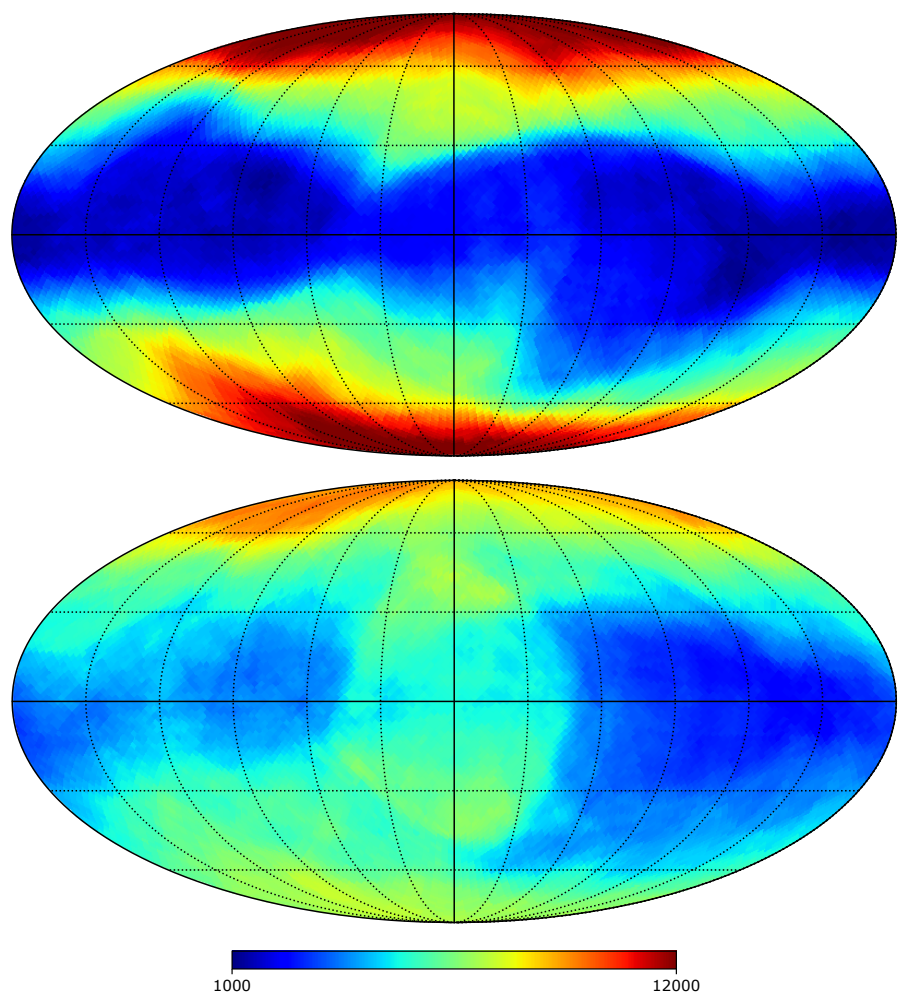

Fig. 2. Map of the exposure time in a one-year simulation by CNES (Jaubert et al. 2017) in kilo-seconds represented in Galactic coordinates (longitude increasing from right to left). Top: nominal phase. Bottom: extended phase.

ECLAIRs. Its detection plane DPIX is composed of $80 \times 80 \mathrm{CdTe}$ pixels of $1 \mathrm{~mm}$ in thickness and $4 \times 4 \mathrm{~mm}^{2}$ active surface each, arranged on a grid with $4.5 \mathrm{~mm}$ spacings between pixels. Its selfsupporting tantalum mask has dimensions of $54 \times 54 \mathrm{~cm}^{2}$ and $0.6 \mathrm{~mm}$ thickness, providing imaging capabilities up to $120 \mathrm{keV}$. With its mask-detector distance of $46 \mathrm{~cm}$, the total field of view is $2 \mathrm{sr}$ and the localisation accuracy of sources at detection limit amounts to about 12 arcmin. The onboard data processing, including the trigger algorithms for GRB detection and localisation, is carried out by the Scientific Trigger and Control Unit, called UGTS in French for Unité de Gestion et de Traitement Scientifique (Schanne et al. 2013; Le Provost et al. 2013), based on an FPGA for data preprocessing for the trigger and a Leon3 dual-core CPU running the complete ECLAIRs flight software including the trigger.

As ECLAIRs' tantalum mask is opaque to soft gamma-ray photons, a source emitting such photons will project the shadow of the mask pattern onto the detector, and the image recorded by the detector is called a shadowgram. The sky image is reconstructed from the shadowgram using the mask deconvolution method (as is currently used by the IBIS telescope onboard the ESA INTEGRAL observatory; see e.g. Goldwurm et al. 2003 for a description of the method). The deconvolution uses the detected number of counts per pixel $D_{\text {cnt }}$ and, assuming a Poissonian distribution per detector pixel $\left(D_{\mathrm{var}}=D_{\mathrm{cnt}}\right)$, it produces reconstructed sky images in number of counts $\left(S_{\mathrm{cnt}}\right)$ and variance $\left(S_{\text {var }}\right)$ from which an signal-to-noise $(\mathrm{S} / \mathrm{N})$ sky image is computed: $S_{\mathrm{S} / \mathrm{N}}=S_{\mathrm{cnt}} / \sqrt{S_{\mathrm{var}}}$.

To detect GRBs with the ECLAIRs telescope, the UGTS uses two different simultaneously running trigger algorithms. A count-rate trigger monitors significant rate increases on timescales from $10 \mathrm{~ms}$ up to $20.48 \mathrm{~s}$ followed by the imaging 


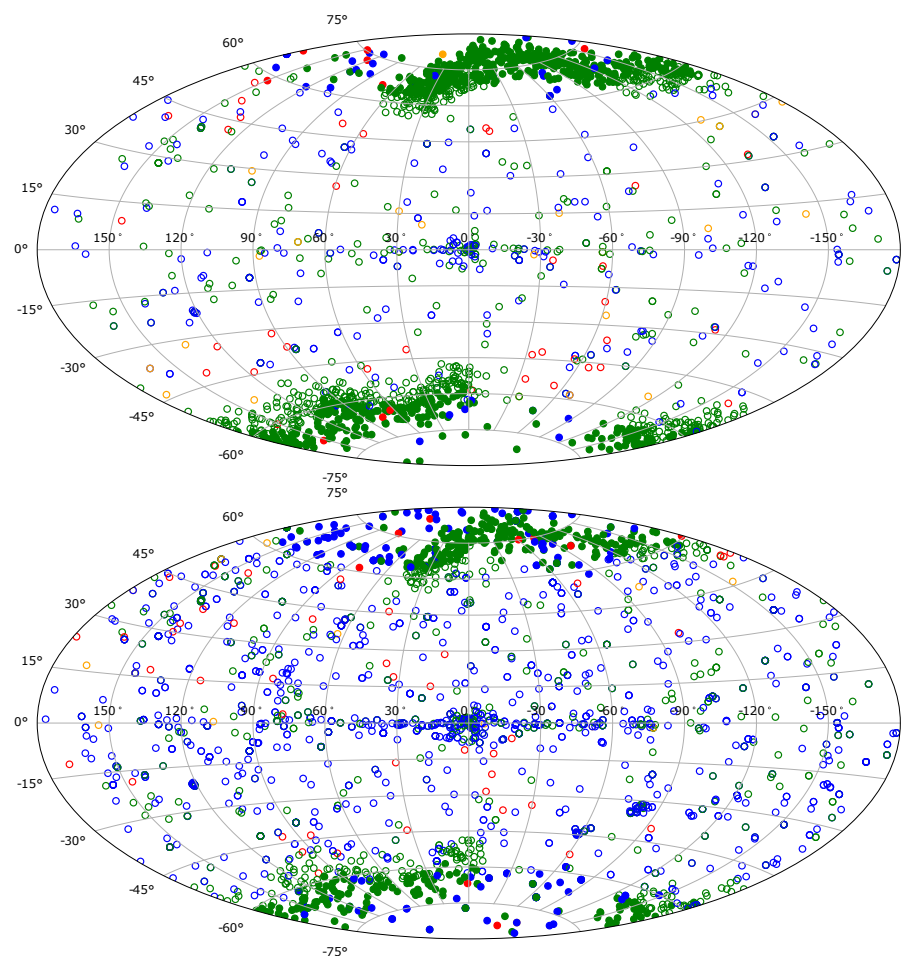

Fig. 3. Distribution of the pointing directions on the sky in Galactic coordinates in a one-year simulation by CNES (Jaubert et al. 2017) Top: nominal phase. Bottom: extended phase. Filled circles show pointings inside the B1 law, empty circles show pointings outside the B1 law. Green is used to signify general program pointings, blue shows nominal targets of opportunity (transient follow-up, GRB revisits), orange shows exceptional targets of opportunity (exceptional astronomical events requiring fast follow-up), and red signifies GRB follow-up pointings.

of the sky in the time window of the excess. It is designed to detect short GRBs or long GRBs with short spikes. An image trigger builds sky images from $20.48 \mathrm{~s}$ of exposure, which are stacked to systematically form sky images on timescales from $20.48 \mathrm{~s}$ up to $\sim 20 \mathrm{~min}$, suited for the detection of long and ultralong GRBs (Dagoneau et al. 2020). These algorithms are implemented in the UGTS (see Fig. 4, Schanne et al. 2015, 2019). The trigger algorithms are foreseen to run in four adjustable energy bands, currently set to: 4-20, 4-50, 4-120, 20-120 keV.

The ECLAIRs telescope is affected by different background components (Zhao et al. 2012; Mate et al. 2019): the cosmic $\mathrm{X}$-ray background (CXB) composed of photons from unresolved extra-galactic X-ray sources plus the reflection of the CXB on the Earth's atmosphere and the intrinsic emission of the atmosphere (albedo) caused by the Earth passages in front of the field of view. We only take into account the CXB while ignoring its reflection and the Earth albedo, because in this study we do not consider the possible presence of the Earth in the field of view of ECLAIRs (and because the CXB dominates the background even in cases where Earth appears in the filed of view). In ECLAIRs, due to the geometry and the large field of view of the instrument, the CXB produces a non-flat close-to-quadratic shape on the detection plane that needs to be removed prior to the deconvolution (see Sect. 3.5) in order to ensure the detection of GRBs with a low false-alarm rate and a good sensitivity to faint events. In addition, some known X-ray sources will affect the onboard trigger performances and can be considered as background components.
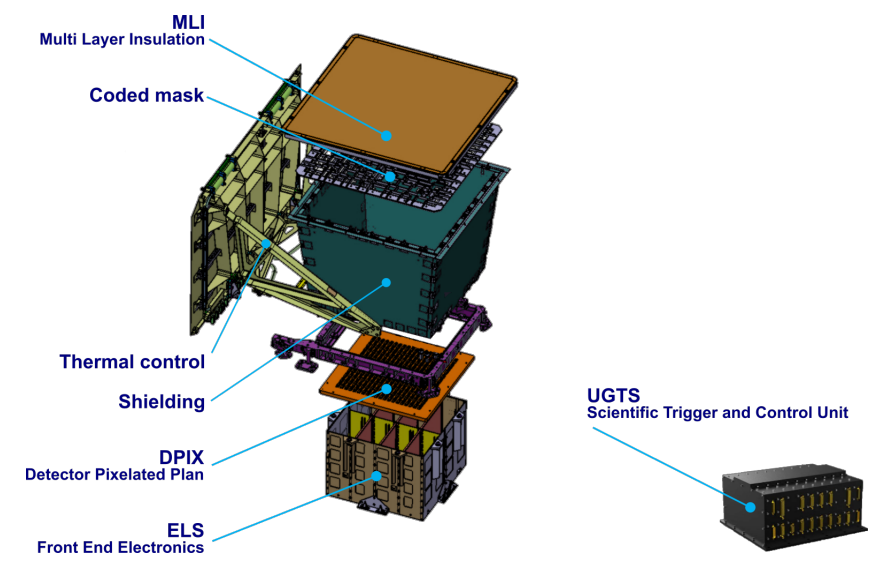

Fig. 4. Schematic representation of the ECLAIRs telescope onboard SVOM.

Because of the large field of view of ECLAIRs ( $2 \mathrm{sr})$, even if the pointing direction of the spacecraft respects the B1 law (see Sect. 1) most of the time, this cannot be guaranteed during the GRB follow-up after a slew or during a part of the general program or target-of-opportunity observations. As an example, if ECLAIRs points towards a position respecting the B1 law (a Galactic pole for instance), and then a GRB is detected, a slew is performed by the spacecraft and it is very likely that parts of the Galactic plane or bulge, and therefore bright known sources, will enter the field of view. During the long-GRB follow-up observation (up to 14 orbits), the bright sources will disturb the trigger algorithms in their search for another GRB or a source outbreak. Also, during general program observations that may occur outside the B1 law, the GRB triggering system is enabled. Therefore, some known X-ray sources can be present in the field of view, in particular bright XRBs (such as Sco X-1, Cygnus X-1) or other bright X-ray sources (pulsar wind nebula and/or pulsars, such as the Crab) that are mostly located in the Galactic plane and bulge.

In the previously described situation, which will represent a large fraction of the overall observing time of ECLAIRs, the onboard trigger algorithms have to deal with the presence of known X-ray sources in the field of view of ECLAIRs that reduce the GRB-detection efficiency. Indeed, the known X-ray sources are targets of interest for the SVOM general program but may be considered as sources of noise for the core program and the detection of GRBs by ECLAIRs' triggering system. The known sources in the field of view of ECLAIRs lead to the superposition of different shadows in its detector-shadowgram and to coding noise after deconvolution. In order to enhance the GRB-detection capabilities, bright known sources have to be "cleaned", that is, their influence must be reduced in the image-reconstruction process. Thus, ECLAIRs' onboard software needs a catalogue of known X-ray sources in order to automatically define the source-cleaning strategy and source avoidance during the GRB searches. The detailed cleaning methods used and their performances will be presented in a future paper. In the following section, we present the way the catalogue is built, including how we generate spectra in order to be able to project the source through a simulator of the ECLAIRs telescope. In Sect. 3 we show the influence of the known Xray sources on ECLAIRs' noise level and we introduce the way sources will be processed by the onboard computer, and in Sect. 3.5 the different methods that can be used to clean their contributions. The structure of the onboard catalogue is described in Sect. 4. 
Table 1. X-ray wide-field instruments (table built with inputs from Krimm et al. 2013).

\begin{tabular}{lcccc}
\hline \hline $\begin{array}{l}\text { Mission } \\
\text { instrument }\end{array}$ & $\begin{array}{c}\text { Period of } \\
\text { operation }\end{array}$ & $\begin{array}{c}\text { Energy range } \\
(\mathrm{keV})\end{array}$ & $\begin{array}{c}\text { Source localisation } \\
\text { accuracy }\end{array}$ & $\begin{array}{c}20 \text { min sensitivity } \\
(3 \sigma ; \text { mCrab })\end{array}$ \\
\hline CGRO/BATSE & $1991-2000$ & $20-1800$ & $>0.2^{\circ}$ & 637 \\
RXTE/ASM & $1995-2012$ & $2-12$ & 5 arcmin & 127 \\
Swift/BAT & $2004-\ldots$ & $14-195$ & $2.5 \operatorname{arcmin}(1 \sigma)$ & 136 \\
Fermi/GBM & $2008-\ldots$ & $8-500$ & $\approx 0.5^{\circ}(b)$ & 1274 \\
MAXI/GSC & $2009-\ldots$ & $2-20$ & $1.5^{\circ}$ & 76 \\
\hline
\end{tabular}

Notes. ${ }^{(a)}$ Assuming the sensitivity is proportional to $\sqrt{t}$, where $t$ is the observing time. ${ }^{(b)}$ The Fermi/GBM localisation accuracy of $\approx 0.5^{\circ}$ corresponds to the one achieved for the monitoring of a catalogue of bright sources with the Earth occultation technique (Wilson-Hodge et al. 2012). The localisation accuracy is larger for the GRB detection: $3.7^{\circ}$ for $90 \%$ of the bursts extending up to $\approx 14^{\circ}$ (Connaughton et al. 2015).

\section{Catalogue of sources}

\subsection{Inputs}

We need to build a list of known X-ray sources that may be seen by ECLAIRs in an observation of $\sim 20$ min duration, which corresponds to the longest exposure time currently foreseen onboard (stacking of 64 sky images with exposures of 20.48 s). Longer exposure mosaics are expected to be constructed on ground by an offline scientific analysis software. The catalogues of sources needed as input to this offline software are developed by the team in charge of the ground pipelines and are not discussed in this article. To build the list of sources for the onboard catalogue, we selected all high-energy sources detected by active wide-field/all-sky monitors. Table 1 gives the list of former and active $\mathrm{X}$-ray wide-field instruments.

We need to build a catalogue of sources that allows us to estimate the count rate of each source in the various energy bands used by ECLAIRs' onboard software. As a full spectral analysis is beyond our requirements, we use the data from both MAXI/GSC (2-20 keV) and Swift/BAT (15-150 keV) as input, which best cover the energy range of ECLAIRs.

\subsubsection{Data for MAXI/GSC sources}

For MAXI/GSC (Matsuoka et al. 2009) we downloaded the standard data products ${ }^{1}$ for all of the 425 sources in the MAXI list (as of 2019-11-06), including daily binned light curves in photons $/ \mathrm{s} / \mathrm{cm}^{2}$ in three energy bands $(2-4,4-10,10-20 \mathrm{keV})$ with associated statistical errors. As an example, we show in Fig. 5 the light curves of the XRB Centaurus X-3 (Cen X-3) obtained in the three MAXI/GSC bands.

\subsubsection{Data for Swift/BAT sources}

For Swift/BAT, we use the BAT 105 months catalogue (Oh et al. 2018) which contains 1632 sources, with source fluxes in units of counts/s/pixel given in eight energy bands covering 14$195 \mathrm{keV}$. The fluxes are extracted from BAT maps built over 105 months and statistical errors are given (hence there is no light curve in the eight bands). The fluxes are converted to photons $/ \mathrm{s} / \mathrm{cm}^{2}$ using the measured Crab fluxes in the eight bands given by Baumgartner et al. (2013) (Table 2) and assuming a Crab powerlaw spectrum with index -2.15 and normalisation $10.17 \mathrm{ph} \mathrm{s}^{-1} \mathrm{~cm}^{-2} \mathrm{keV}^{-1}$ (Baumgartner et al. 2013). The catalogue also provides a photon powerlaw index over the eight

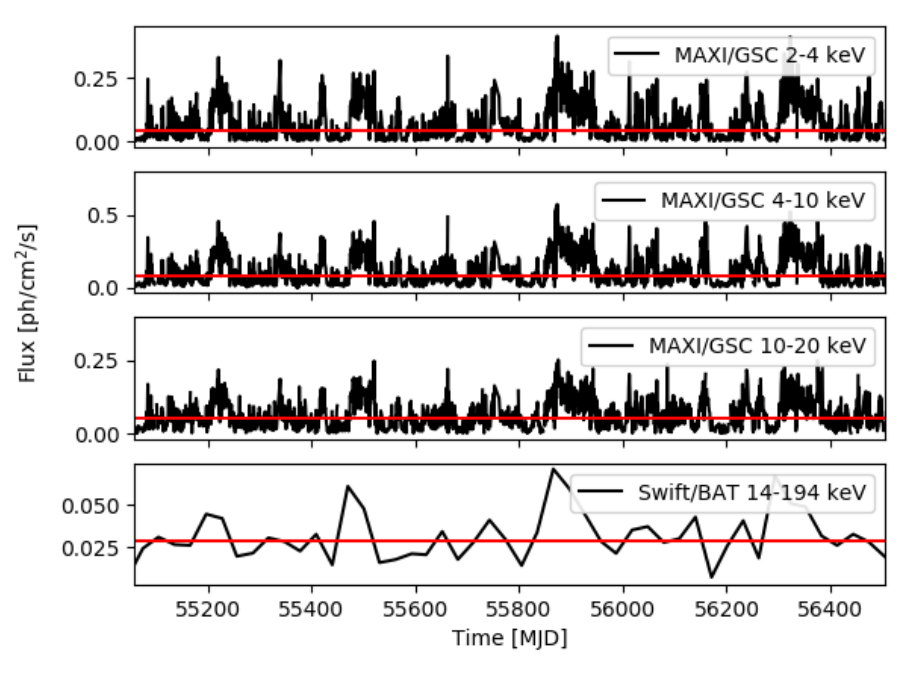

Fig. 5. Examples of light curves for the source Cen $X-3$ in the three MAXI/GSC bands and in the total Swift/BAT band. The red lines indicate the median flux over the light curve in each energy band for MAXI, and the median of the sum of the fluxes in eight energy bands for Swift/BAT.

bands. As an example, we show in Fig. 5 the light curve of the XRB Cen X-3 over the complete Swift/BAT energy range. The red line gives the median of the sum of the fluxes in the eight bands.

\subsection{Cross-correlation of catalogues and celestial position}

As the MAXI/GSC and Swift/BAT instruments provide crude positions (in comparison to the ones given by focused instruments such as Chandra or XMM-Newton in X-rays or even optical positions of their counterparts when they are known), the source positions that will be given in the onboard catalogue are the best positions that are reported in SIMBAD (Wenger et al. 2000). Most of these come from the Gaia data release Gaia Collaboration (2018).

Many sources have various names or identifiers, often associated with different counterparts in various spectral windows. In order to associate a MAXI source to a Swift one (and vice versa) we use the Sesame name resolver ${ }^{2}$ for each source to find the main identifier. For instance, Crab has 56 identifiers (M 1, Taurus A, Swift J0534.6+2204, etc.). Its main identifier is M 1. A few sources are not found by the Sesame name resolver and 
hence are not associated between MAXI/GSC and Swift/BAT and in these cases we use the instrumental position. Over the 425 sources from MAXI/GSC, 264 have a counterpart in the Swift/BAT 105 months catalogue. Sources with no counterpart in one of the two input catalogues are added to the list. This leads to a list of 1793 sources.

The Swift/BAT team, besides providing the 105 months catalogue, also provides a list of sources for the BAT hard X-ray transient monitor (Krimm et al. 2013). This list contains 1026 sources but only 305 are considered as detected by BAT. From these 305 sources, some are already included in the list (from MAXI and/or Swift side). There are 44 sources that are not present either in the MAXI/GSC list or in the Swift/BAT 105 months catalogue. However, these sources have either a large excess variance $\left(F_{\mathrm{var}}>3\right)$ or a mean square error larger than the intrinsic sample variance. According to Krimm et al. (2013) they are classified as flaring or outburst sources. This means that they exhibit a high flux over a short time. Such sources should not be considered as disturbing sources for the detection of GRBs and are even sources of interest if detected. This is why we do not project them through ECLAIRs' simulator, as they are not really considered as background sources. If an outburst of such a source is detected onboard, the source will be identified on ground with a search in various catalogues (see Sect. 4.2).

Finally, our list contains 1793 sources (i.e. we found 264 sources present in both MAXI/GSC and Swift/BAT catalogues, which are considered only once in our list of sources). However, it is very likely that ECLAIRs will not detect all of theses sources, even on its longest timescale of $20 \mathrm{~min}$. Based on ECLAIRs' sensitivity, which is determined in Sect. 3.1, and on the source spectra (see Sect. 2.4), 53 sources have a flux above the sensitivity limit in the fully coded field of view in 20 min and in at least one of ECLAIRs' four energy bands. In $20 \mathrm{~s}$ the number drops to 12 sources. Table A.1 lists the sources we determined to be bright enough to be detectable by ECLAIRs in $20 \mathrm{~min}$ (based on their "typical S/N", see Sects. 3.1 and 3.4 for the presentation of the selection criteria for this catalogue).

\subsection{Other X-ray sources}

Besides the sources included from the MAXI/GSC source list or from the Swift/BAT 105 months catalogue, we also investigate whether or not our list of sources contains other X-ray sources such as magnetars or type I X-ray bursters. For the magnetars, we cross-match our list of 1793 sources with the list of 30 known magnetars from Olausen \& Kaspi (2014). We find that 12 magnetars out of the 30 are included in our list (with XTE J1810-197 among the brightest sources). Concerning the type I X-ray bursters, we cross-match our list with the Multi-INstrument Burst ARchive (Galloway et al. 2020) containing 115 bursts from 85 sources. From this catalogue, 32 sources are missing from our list. Considering the missing magnetars and type I X-ray bursters (50 sources), we do not add them to our list because they are faint sources (as they are not reported in the MAXI/GSC or Swift/BAT lists). In the case where the ECLAIRs triggering system detects one of these sources, it will be considered as a GRB candidate and the true identification will be found on ground after a search in catalogues around that position. Newly detected sources (GRB candidates) are temporally included in the onboard catalogue in order to prevent re-tirggering on them after a first trigger. If desired, typically every week, sources in the onboard catalogue can be removed (or added) by telecommand.

\subsection{Spectra}

Now that we have a list of sources, we can generate the spectra for all 1793 sources using the MAXI/GSC and/or Swift/BAT 105 months data. Spectra are needed to be able to simulate the sources in ECLAIRs' model, and to evaluate their influence on the background level (see Sect. 3). To build those spectra, we proceed as follows.

1. If the source has MAXI/GSC and Swift/BAT data, a joint broadband fit is performed with a single powerlaw (Eq. (1)) and a broken powerlaw (Eq. (2)):

$$
\begin{aligned}
& N(E)=K \cdot E^{\alpha}, \\
& N(E)= \begin{cases}K \cdot E^{\alpha} & \text { if } E \leq E_{\text {break }} \\
K \cdot E^{\beta} \cdot E_{\text {break }}^{\alpha-\beta} & \text { if } E>E_{\text {break }},\end{cases}
\end{aligned}
$$

where $N(E)$ is the photon flux density (i.e. the number of photons per unit energy bandwidth per unit area per second), $K$ stands for the spectrum normalisation (photons $/ \mathrm{cm}^{2} / \mathrm{s} / \mathrm{keV}$ at $1 \mathrm{keV}$ ), and $\alpha$ and $\beta$ are the photon indexes. In the case of a broken powerlaw, $E_{\text {break }}$ is the break energy at which the slope changes. The fitted fluxes are the median fluxes (preferred to the mean, in order to get a flux that is less dependent on potential outbreaks of faint sources) of the three MAXI/GSC sub-band light curves plus the fluxes of the eight Swift/BAT sub-bands (given in the source pulse height analyser file). The energy corresponding to each flux point is set to the centre of its sub-band. Out of both performed fits, we choose to keep the powerlaw (instead of the broken powerlaw) if one of the following is true:

- the summed squared error of the single powerlaw is smaller than that of the broken powerlaw;

- the break energy is below $8 \mathrm{keV}$ or above $80 \mathrm{keV}$;

- the slope is steeper at low energy than at high energy $(|\alpha|>$ $\beta \mid)$;

- the difference between the two photon indexes of the broken powerlaw is smaller than 1 (this leads to an error on the flux of $10 \%$ or less for the 17 sources affected by this constraint).

2. If the source has only MAXI/GSC data, a fit is performed with a single powerlaw (Eq. (1)). The fitted fluxes are the median fluxes in the three MAXI/GSC sub-band light curves.

3. If the source has only Swift/BAT data, the spectrum is the single powerlaw (Eq. (1)) given by the BAT 105 month catalogue (Oh et al. 2018).

In a last step, if a source is detected by MAXI/GSC, its spectrum normalisation is corrected to ensure that the integration of our spectrum reaches the median flux of MAXI/GSC in $4-20 \mathrm{keV}$. This allows us to correct for a possible offset between BAT and GSC spectra. This offset may arise after fitting the fluxes computed from different time windows in the two instruments for sources that are known to vary within typical scales of days or months.

The absorption is not taken into account since ECLAIRs will operate above $4 \mathrm{keV}$ and all sources in the catalogue have an HI column density $N_{\mathrm{H}}<2.27 \times 10^{22}$ atoms $\mathrm{cm}^{-2}$ with a mean of $2.63 \times 10^{21}$ atoms cm $\mathrm{cm}^{-2}$ (see Sect. 2.5). Compared to a nonabsorbed powerlaw ( $\alpha=-2, K=10$ ), taking into account this mean $N_{\mathrm{H}}$ value reduces the flux by $0.6 \%$ in $4-20 \mathrm{keV}$ (the influence is even smaller on larger energy bands).

In the case of the well-known Crab, this procedure leads to the reconstructed spectrum shown in Fig. 6. We find the best model is a single power law with a normalisation $K=$ $11.2 \pm 1.1 \mathrm{ph} \mathrm{s}^{-1} \mathrm{~cm}^{-2} \mathrm{keV}^{-1}$ (at $1 \mathrm{keV}, 1 \sigma$ ) and a photon index 


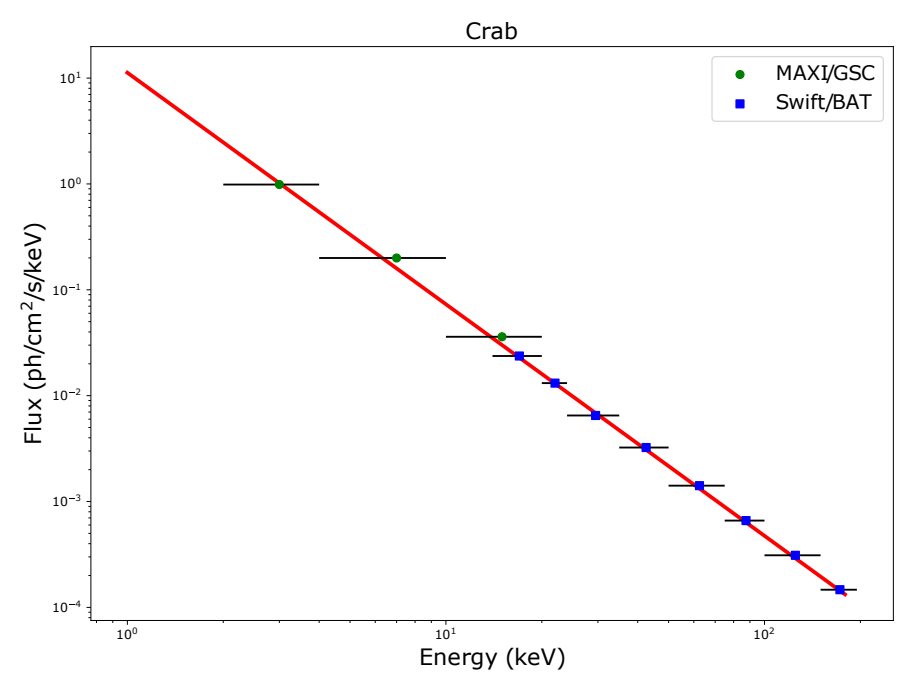

Fig. 6. Example spectrum for Crab obtained using our procedure.

$\alpha=-2.19 \pm 0.03(1 \sigma)$. This result is very close to the one usually reported in the literature (Toor \& Seward 1974; Weisskopf et al. 2010).

Figure 7 gives an example of a spectrum for the source Hercules X-1 (Her X-1), whose best-fit model is a broken power law with $\alpha=-0.72 \pm 0.21(1 \sigma), \beta=-4.09 \pm 0.18(1 \sigma)$ and $E_{\text {break }}=20.04 \pm 0.46 \mathrm{keV}(1 \sigma)$. These values agree with the ones from dal Fiume et al. (1998). However, we note that these authors have a best-fit model consisting of an exponentially cutoff powerlaw with $\alpha=-0.884 \pm 0.003$ (90\% C.L.) and a cut-off energy of $24.2 \pm 0.2 \mathrm{keV}$. We also estimate an approximate slope of -4.2 from the spectra shown in their Fig. 1 (right).

We note that the reduced $\chi^{2}$ are large $\left(3.8 \cdot 10^{4}\right.$ for $\mathrm{Crab}$ and $6.3 \times 10^{2}$ for Her X-1) which may be caused by the fact that the sources are bright and have small statistical errors and also by the coarse energy binning ${ }^{3}$. This effect has already been observed by Baumgartner et al. (2013) for the fit of BAT spectra with a simple power law. We note that systematic errors are not taken into account.

Also, some sources are known to exhibit some spectral changes (e.g. Sco X-1, GRS 1915+105, Cyg X-3, Cyg X-1, etc.). These changes are related to different behaviours of the accretion disc around the compact object in XRBs. In our study, as the spectra are built over a long observational timescales, the best models we obtain for these sources do not represent one particular state. We reiterate the fact that we only need spectra to be able to evaluate the influence of the sources on the background level of ECLAIRs and a detailed spectral analysis is beyond our requirements. Moreover, the bright sources will be cleaned regardless of their states (see Sect. 3.5).

As a result, 1698 sources can be well modelled with a single power law spectrum and 95 sources are described by a broken power law. Table A.1 also gives the spectral parameters of the brightest sources. The full table giving the general properties, the data in the spectral bands from MAXI/GSC and Swift/BAT, and the spectral fit we performed, for all the 1793 sources, is available at the CDS.

\footnotetext{
3 The flux in an energy bin is distributed across the entire bin width with a specific law and not just in its centre as we assumed. Therefore, the error on the flux in the centre of the bin depends on the statistical error and on a systematic error based on the bin width. Taking this error into account should help to reduce the $\chi^{2}$.
}

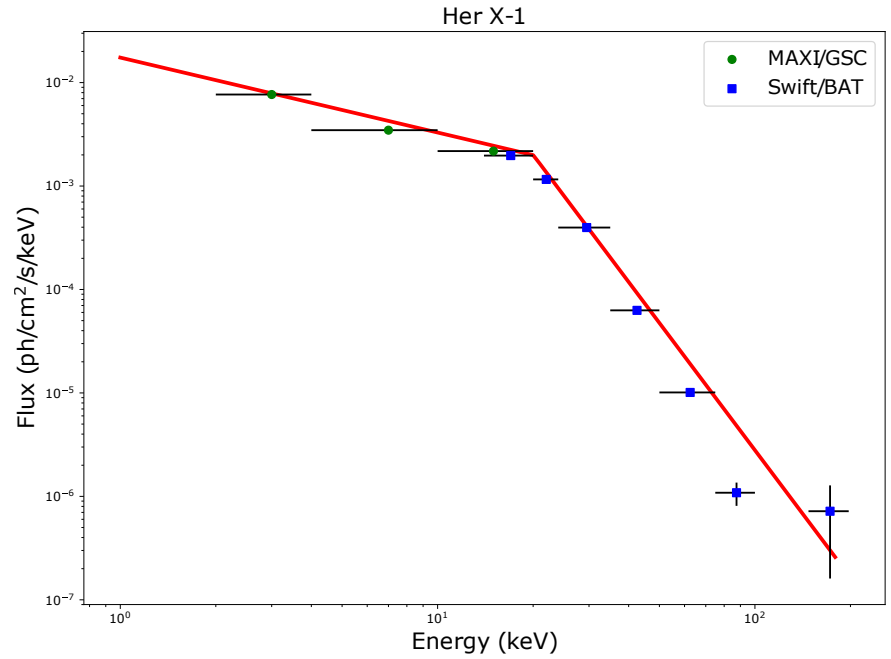

Fig. 7. Example spectrum for Her X-1 obtained using our procedure.

In the simulations presented in Sect. 3, the sources will be projected through ECLAIRs' model, using the photon flux obtained by the integration of the spectrum in a maximum energy range of 4 to $150 \mathrm{keV}$. Photons from sources without Swift/BAT counterpart will be rejected above $20 \mathrm{keV}$, while photons from sources without MAXI/GSC counterpart (present only in the BAT 105 month catalogue) will be rejected below $14 \mathrm{keV}$. The sources only present in the Swift/BAT hard X-ray transient monitor list will not be projected.

\subsection{Population statistics}

In this section we provide some statistics on the catalogue we have built. First, the Fig. 8 shows the distribution of the sources in the sky. The plain circles, which depict the 53 brightest sources of the catalogue that ECLAIRs should be able to detect in $20 \mathrm{~min}$ images (according to its sensitivity, see Sect. 3.1), are clustered in the Galactic plane. These sources are mostly accreting XRBs with either a neutron star or a black hole as the primary compact object.

Figure 9 gives the distributions of the flux in the $4-120 \mathrm{keV}$ spectral range and the ones of the spectral parameters obtained from the fits to our reconstructed spectra (Sect. 2.4): $\alpha, \beta$ and $E_{\text {break }}$. The mean value of the powerlaw index is $\alpha=$ $-2.01 \pm 0.65(1 \sigma)$. In the break energy histogram (Fig. 9 bottom right), two groups are visible at $E_{\text {break }} \sim 10 \mathrm{keV}$ and $E_{\text {break }} \sim$ $18 \mathrm{keV}$. The latter corresponds to breaks between MAXI/GSC and Swift/BAT energy bins and is consistent with a broken powerlaw (see Fig. 7). The former group has a break value only within the MAXI/GSC spectral range. In this population, the low value of the energy break could, in part, phenomenologically mimic the effect of absorption that we have not taken into account. The absorption indeed has a stronger effect on the low energy photons and therefore tends to bend the low part of the spectra towards smaller values. The resultant spectra are therefore harder: their photon index is smaller (in absolute value) below the energy where the absorption no longer affects the radiation (taken as $E_{\text {break }}$ ). Figure 10 shows the distribution of the hydrogen column density at the sources' position.

Table 2 gives a summary of the minimum, maximum, mean and standard deviation of each of the previous values.

Table 3 gives the source types obtained from the BAT 105 months catalogue (Oh et al. 2018), or from the Simbad database 

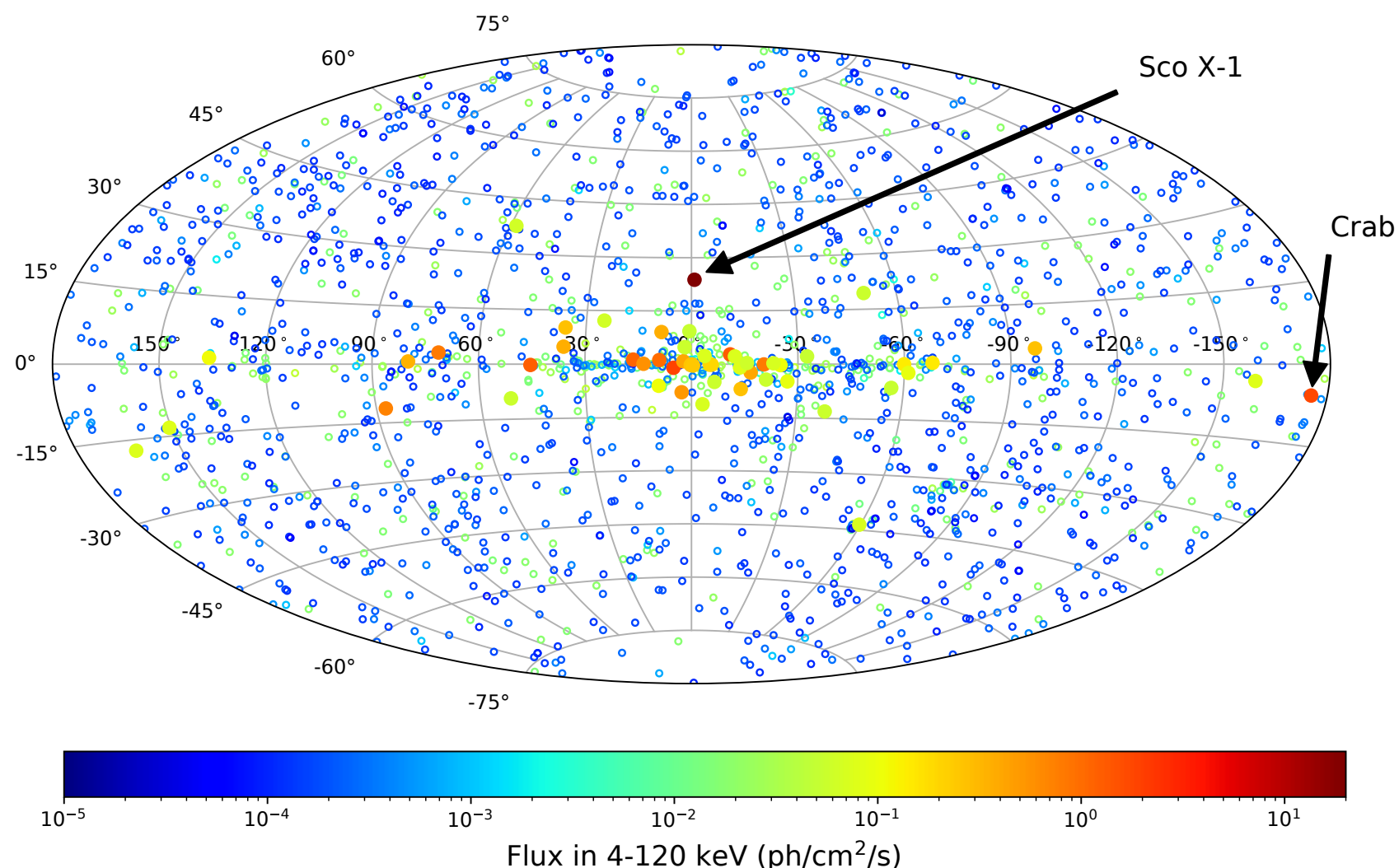

Fig. 8. Distribution of the sources of our catalogue on the sky in Galactic coordinates. Empty circles correspond to the full catalogue and plain circles correspond to the sources that should be visible in $20 \mathrm{~min}$ according to the expected sensitivity of ECLAIRs. The colour gives the flux in $4-120 \mathrm{keV}$ in $\mathrm{ph} / \mathrm{cm}^{2} / \mathrm{s}$.
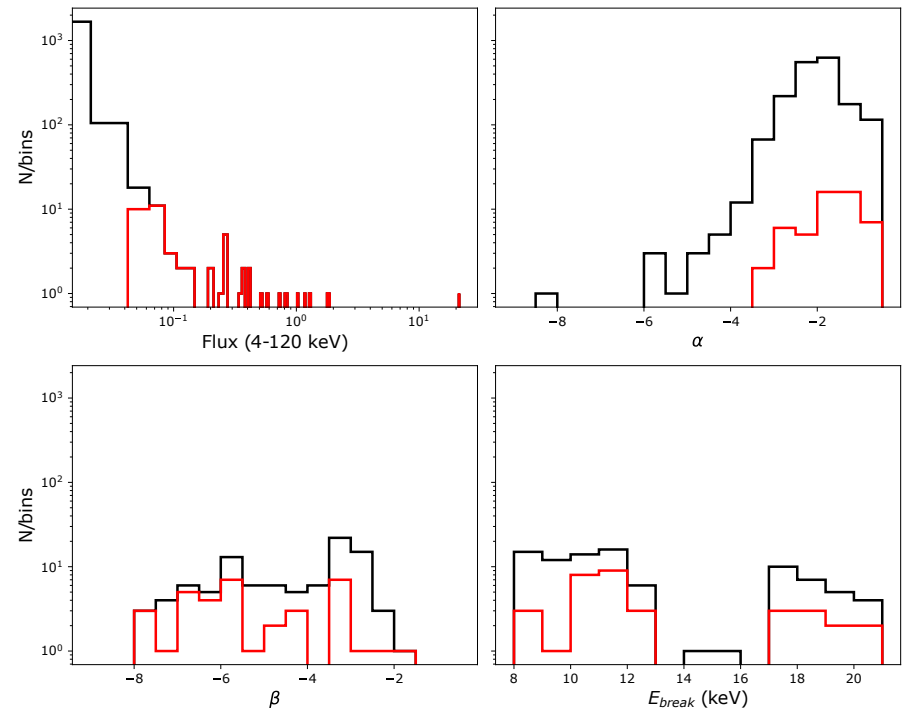

Fig. 9. Distribution of the spectral parameters. Top left: 4-120 keV flux $\left(\mathrm{ph} / \mathrm{cm}^{2} / \mathrm{s}\right.$ ). Top right: photon index $\alpha$. Bottom left: photon index $\beta$ (for the sources with a broken power-law spectrum). Bottom right: break energy in keV: $E_{\text {break }}$ (for the sources with a broken power-law spectrum). Black histograms represent the full catalogue, whereas red histograms represent the brightest 53 sources.

(Wenger et al. 2000) if unknown from BAT, for the full catalogue and for the 53 brightest sources (according to the sensitivity, see Sect. 3.1). Most of the sources in the catalogue are active galaxies or XRBs. The brightest sources are mainly low-mass XRBs.

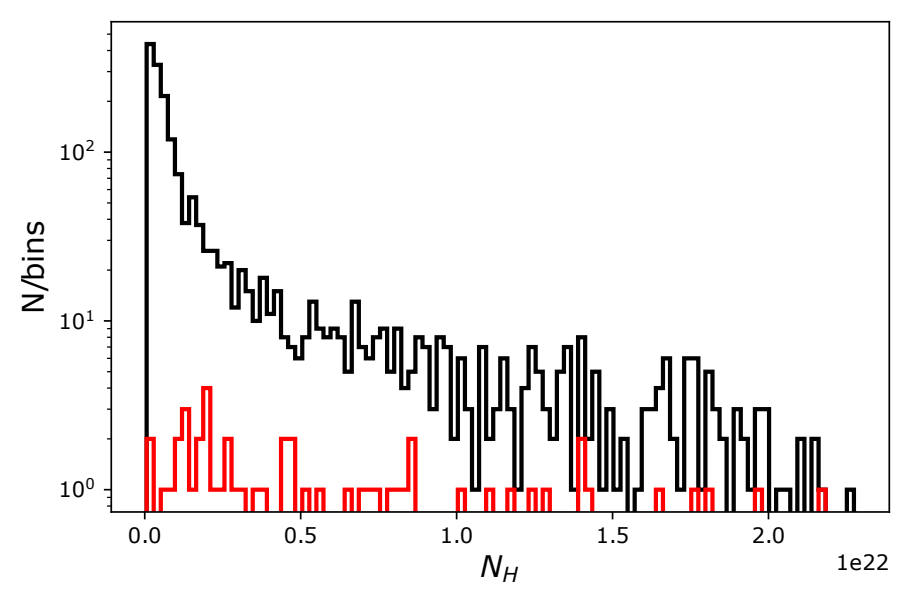

Fig. 10. Distribution of the hydrogen column density (in units of $10^{22}$ atoms cm$~_{-2}$ ) $N_{\mathrm{H}}$ at the source positions retrieved from Bekhti et al. (2016). Black: full catalogue. Red: brightest 53 sources.

\section{Simulations through ECLAIRs}

In this section, we present the simulations performed in order to estimate the influence of the catalogue sources on ECLAIRs' background level. First we determine the sensitivity of ECLAIRs with respect to the sources in Sect. 3.1, and then we simulate the sources through ECLAIRs' model in Sect. 3.2. For the different simulations, we use our ray-tracing simulation software, which propagates photons one by one through ECLAIRs' coded mask. The ray-tracing takes into account the energy redistribution which includes the transparency of the mask and the 
Table 2. Summary of the catalogue statistics.

\begin{tabular}{lcccccc}
\hline \hline & & $F_{4-120}\left(\mathrm{ph} \mathrm{cm}^{-2} \mathrm{~s}^{-1}\right)$ & $\alpha$ & $\beta$ & $E_{\text {break }}(\mathrm{keV})$ & $N_{\mathrm{H}}\left(\mathrm{atoms} \mathrm{cm}^{-2}\right)$ \\
\hline All sources & $\min$ & 0.00 & -8.25 & -7.74 & 8.05 & $6.08 \mathrm{e}+19$ \\
& $\max$ & 21.16 & -0.02 & -1.87 & 22.00 & $2.27 \mathrm{e}+22$ \\
& mean & 0.03 & -2.01 & -4.46 & 13.11 & $2.63 \mathrm{e}+21$ \\
& std & 0.51 & 0.65 & 1.58 & 4.18 & $4.31 \mathrm{e}+21$ \\
\hline Brightest 53 sources & $\min$ & 0.05 & -3.17 & -7.74 & 8.10 & $1.54 \mathrm{e}+20$ \\
& $\max$ & 21.16 & -0.33 & -1.87 & 21.47 & $2.17 \mathrm{e}+22$ \\
& mean & 0.74 & -1.61 & -5.18 & 13.64 & $6.48 \mathrm{e}+21$ \\
& std & 2.87 & 0.60 & 1.66 & 4.15 & $5.64 \mathrm{e}+21$ \\
\hline
\end{tabular}

efficiency of the detector according to the energy of the incident photons. Additionally, in the following simulations, $\mathrm{CXB}$ photons are also propagated through ECLAIRs with a spectrum given by Moretti et al. 2009 (we use the CXB spectrum obtained from Swift/XRT and BAT data in the 1.5-200 keV band described by two smoothly joined power laws with an energy break at $29 \mathrm{keV}$ and two photon indices of 1.40 and 2.88) and a small internal background of 0.003 counts $\mathrm{s}^{-1} \mathrm{~cm}^{-2} \mathrm{keV}^{-1}$ with a flat spectrum is added (Sizun 2011) as an estimated mean contribution of particles over the orbit. In this study we assume that Earth is out of the field of view.

\subsection{Sensitivity of ECLAIRs}

In our catalogue there are 1793 sources detected by MAXI/GSC or Swift/BAT (or both). As MAXI/GSC and Swift/BAT have a better sensitivity than ECLAIRs, many of these sources will be too faint to be seen by ECLAIRs in $20 \mathrm{~min}$. To obtain the subcatalogue of the bright sources that will be seen by ECLAIRs in $20 \mathrm{~min}$, we first need to estimate ECLAIRs' sensitivity in each of the overlapping trigger energy bands ("energy strips") foreseen on board (4-20, 4-50, 4-120, 20-120 keV). To do so, we simulated 5000 dummy sources in the fully coded field of view with uniformly drawn fluence between 0 and $110 \mathrm{ph} \mathrm{cm}^{-2}$ over an exposure time uniformly drawn between $20 \mathrm{~s}$ and $20 \mathrm{~min}$. The maximum fluence of $110 \mathrm{ph} \mathrm{cm}^{-2}$ was chosen in order to fill the fluence-duration plane of our simulation with enough sources to be able to derive the detection sensitivity (over background) close to the maximum duration of $20 \mathrm{~min}$. The dummy sources were ray traced one by one, with the $\mathrm{CXB}$ and the internal background added. The source spectrum is assumed to be a simple power law of index -2 which corresponds to the mean photon index in our catalogue (see Sect. 2.5). The shadowgrams are cleaned before the deconvolution by fitting and subtracting a quadratic-shape model of the CXB. After the cleaning and the deconvolution, the source $\mathrm{S} / \mathrm{N}$ is obtained at the source position in the sky image. For each energy band, a square root law $S=S_{1} \cdot \sqrt{t}$ is fitted ( $S$ denotes the fluence over the observing time $t$, and $S_{1}$ the one-second fluence at the detection limit) using the sources close to a detection threshold of $S / N=6.5$ (Schanne 2009). This value corresponds to the threshold in the $\mathrm{S} / \mathrm{N}$ sky images required to claim the detection of a new source, while ensuring a low false detection rate (less than one per day). The results for the four energy bands are shown in Fig. 11 and Table 4.

The sensitivity and associated errors in the three first bands (which start at $4 \mathrm{keV}$ ) are very close $\left(\approx 1.8 \mathrm{ph} \mathrm{cm}^{-2} \mathrm{~s}^{-1}\right.$, see Table 4 for precise values). When the high-energy bound increases, the ratio between the number of photons of the source
Table 3. Summary of the source types.

\begin{tabular}{lcc}
\hline \hline Type & All sources & Brightest 53 sources \\
\hline AGN & 1127 & 2 \\
Galaxy clusters/groups & 52 & 1 \\
Other galaxies & 9 & 0 \\
\hline HMXB & 135 & 12 \\
LMXB & 132 & 34 \\
Other XRBs & 13 & 0 \\
\hline Stars & 114 & 1 \\
Novæ & 29 & 0 \\
Pulsars & 28 & 1 \\
SNR & 11 & 1 \\
HII/clouds & 4 & 0 \\
Star clusters & 1 & 0 \\
\hline Unknown & 180 & 1 \\
\hline
\end{tabular}

and the number of photons of the $\mathrm{CXB}$ decreases. In other words, the source-to-background relative contribution decreases with energy. For this reason, above $20 \mathrm{keV}$, more photons are required, and therefore a higher fluence, in order to reach the detection threshold. This effect is shown in the bottom-right plot of Fig. 11. The sensitivity therefore drops significantly above $20 \mathrm{keV}$.

We note here that the sensitivity computed with our methods should not be taken as absolute sensitivity values for ECLAIRs, and should therefore be considered with the following caveats. First, our sensitivity values are calculated only in the fully coded field of view $(0.15 \mathrm{sr})$ and they will decrease in the total field of view. Furthermore, they are strongly dependent on the assumed background shape and level. This is why the measured sensitivity during flight operations may differ from the one we give here.

With these calculated sensitivities (Table 4) we can extract the list of the known sources which may be detected in one of the four considered energy bands in a maximum accumulation time of $20 \mathrm{~min}$. This list, or sub-catalogue, is composed of 53 sources and was used in the previous sections to provide some statistics on the brightest sources of the full catalogue. However, even if these crude sensitivities help us estimate the sources that will be seen by ECLAIRs, it cannot replace a complete simulation of all the sources through ECLAIRs (see Sect. 3.4). Such simulation is required to precisely define the list of sources that should be systematically taken into account in the cleaning process and/or blacklisted as known sources for the trigger should they enter in ECLAIRs' field of view during a given observation. 
N. Dagoneau et al.: Onboard catalogue of known X-ray sources for SVOM/ECLAIRs
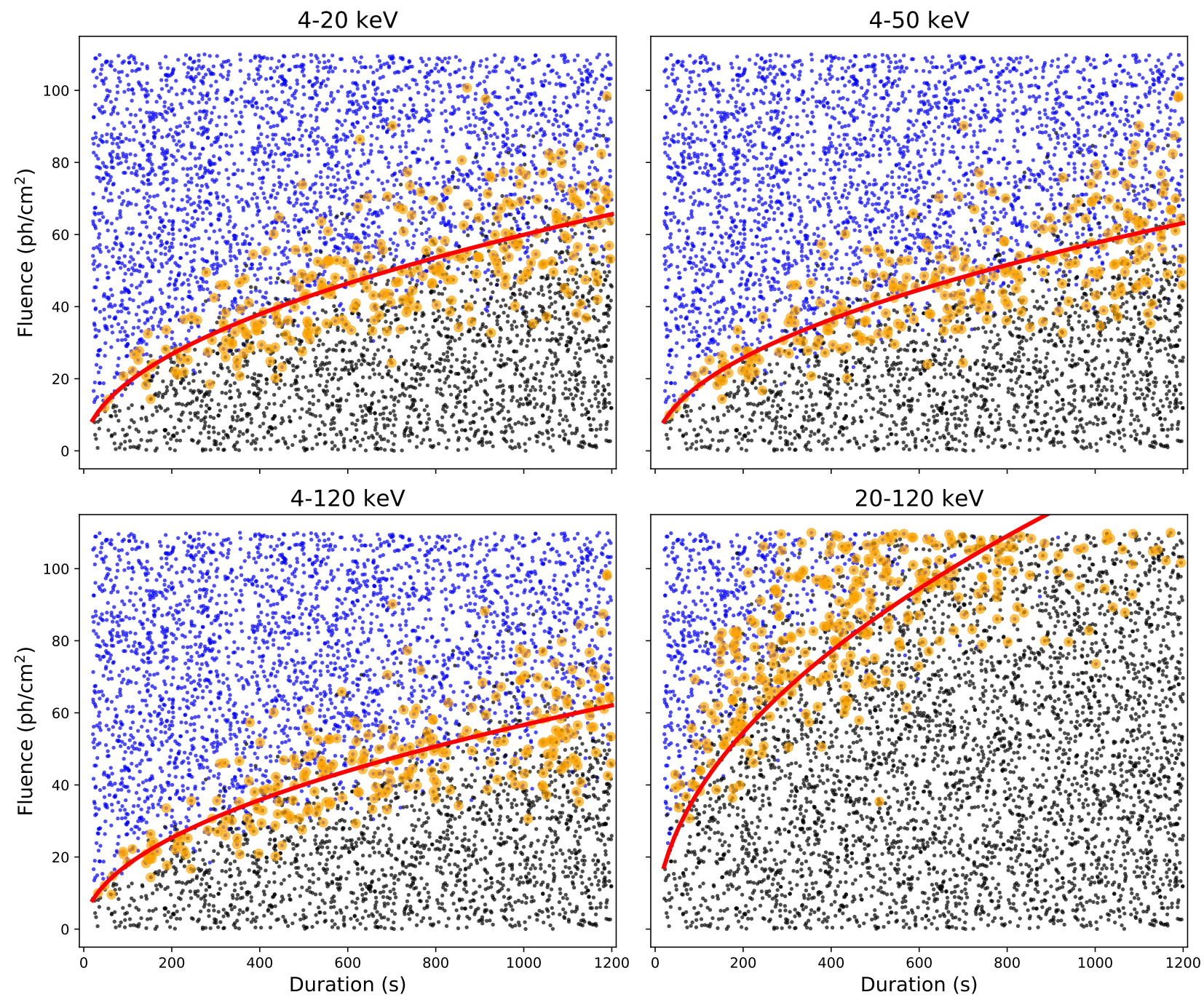

Fig. 11. ECLAIRs' sensitivity in different energy bands according to the exposure time. Blue points indicate sources with $S / N \geqslant 6.5$, and black points indicate sources with $S / N<6.5$. Orange points indicate sources with $6 \leqslant S / N<7$ used to fit the sensitivity law (red curve).

\subsection{Influence of known sources in the detector images before deconvolution}

In order to study the influence of the known X-ray sources on ECLAIRs' noise level, we draw 10000 isotropic pointing positions in the sky (the satellite roll angle is fixed to $0^{\circ}$ ). For each of these pointings, we select the list of sources in the field of view from our full catalogue. Each source is projected by ray tracing from its local coordinates in ECLAIRs' frame. For these simulations, we assume that the sources are persistent at the level of their median flux, even if it is well known that some sources exhibit a variable or bursty behaviour (see Fig. 5). The detailed way the sources are processed and cleaned by the onboard software is described in Sect. 3.5. For each shadowgram (each exposed for $20 \mathrm{~min}$ ) we compute the count rate per second added by the sources in the given field of view. The maps giving this rate are shown in Fig. 12 for the four different energy bands. In these maps, we indicate some sky regions that will be discussed in more detail in the following (see Fig. 8 for precise source location). The central zone where the highest rates are reached corresponds to the Galactic centre and the Sco X-1 region.

In Fig. 12, we first notice that the count rate added by the bright sources is quite variable over the whole sky, and roughly goes from $\approx 100$ counts $^{-1}$ close to the Galactic poles to a
Table 4. Sensitivity of ECLAIRs in different energy bands for detection with $S / N>6.5$.

\begin{tabular}{lcc}
\hline \hline Energy range & $S_{1}\left(\mathrm{ph} \mathrm{cm}^{-2}\right)$ & err $\left(\mathrm{ph} \mathrm{cm}^{-2}, 1 \sigma\right)$ \\
\hline $4-20 \mathrm{keV}$ & 1.895 & 0.026 \\
$4-50 \mathrm{keV}$ & 1.823 & 0.026 \\
$4-120 \mathrm{keV}$ & 1.792 & 0.024 \\
$20-120 \mathrm{keV}$ & 3.855 & 0.045 \\
\hline
\end{tabular}

Notes. Here, $S_{1}$ is the one-second sensitivity and the sensitivity follows $S(t)=S_{1} \cdot \sqrt{t / 1 \mathrm{~s}}$.

maximum of $\approx 5000$ counts $\mathrm{s}^{-1}$ close to the Galactic centre and Sco X-1 (4-120 keV). As a comparison, the CXB contributes to the background level for $\approx 3000$ counts $\mathrm{s}^{-1}$ in $4-120 \mathrm{keV}$. The contribution of known sources is dominant in the first three bands, namely 4-20, 4-50, and 4-120 keV, while in the last band, above $20 \mathrm{keV}$, it is much less important. Because of its broken power-law spectrum and its high-energy photon index of -5.8 above $\approx 10 \mathrm{keV}$, the Sco $\mathrm{X}-1$ contribution fades away in the $20-120 \mathrm{keV}$ band. Table 5 gives the mean number of counts and its standard deviation for the different energy bands 


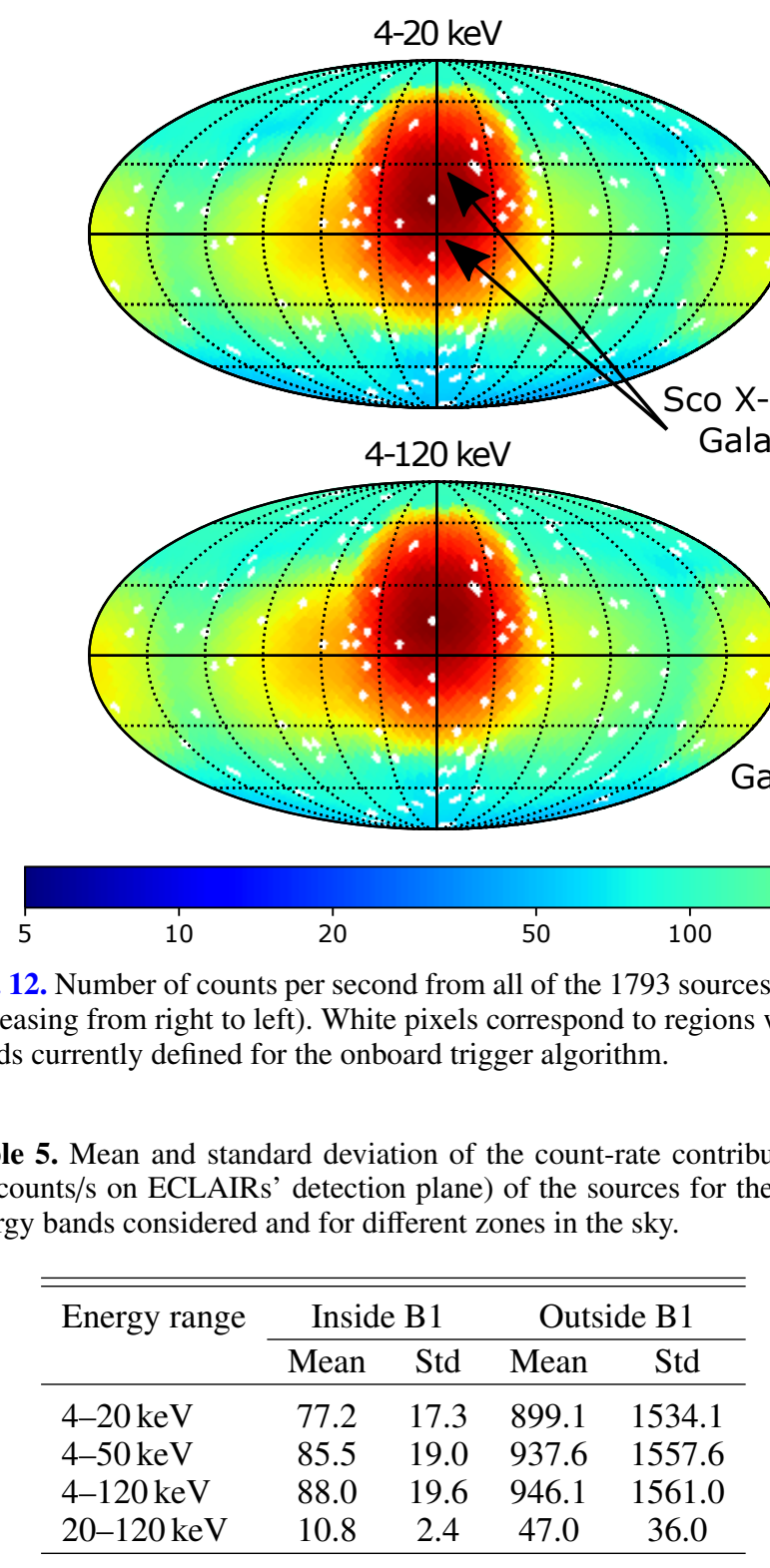

considered and different zones on the sky. The ratio between the mean rate for pointings within the B1 law and outside the B1 law is $\approx 11.1$ for the first three bands and $\approx 4.3$ for the $20-120 \mathrm{keV}$ negligible compared to the $\mathrm{CXB}$, whereas near the Galactic centre and close to Sco X-1, the contribution from known sources is dominant $(\approx 8000$ counts is the Galactic centre). Outside the B1 law, the rate is subject to a large dispersion due to the very different regions within the Galactic plane. The centre is much more crowded with bright sources than the other regions.

\subsection{Influence of known sources on the sky images after deconvolution}

To estimate the effect of bright sources on the deconvolved sky images, we used the same 10000 pointing positions in the sky as the ones used in the previous section. For each of these positions, we generate 64 shadowgrams of $20.48 \mathrm{~s}$ each, built from the contribution of all our catalogue sources in the field of view, with added CXB and internal background contributions. The 64 shadowgrams are deconvolved and the 64 sky images (each

Table 5. Mean and standard deviation of the count-rate contributions
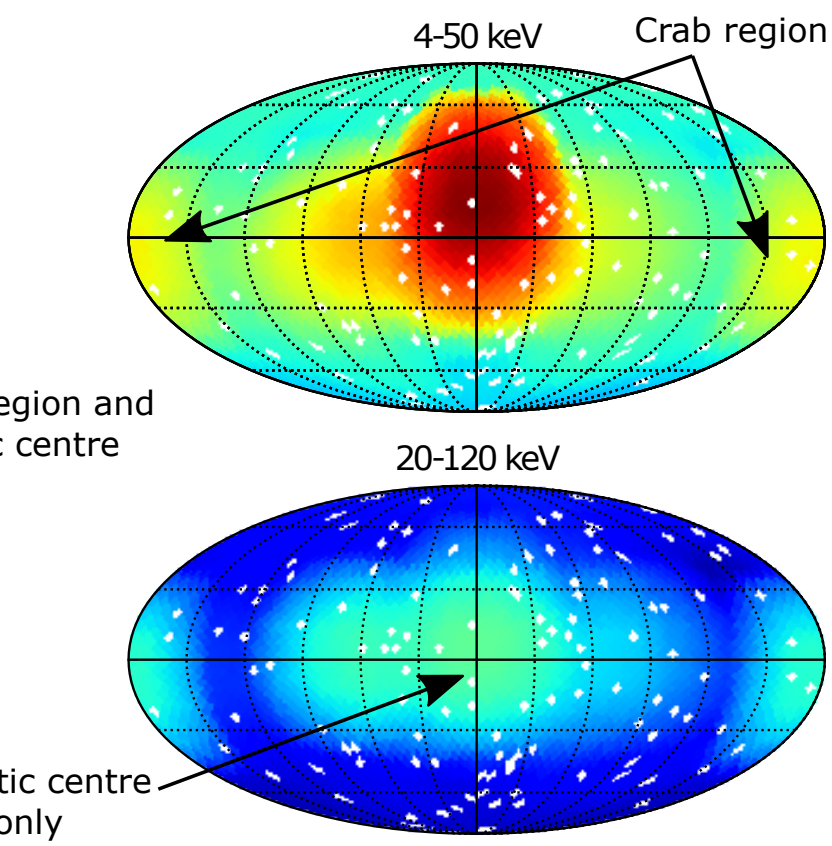
only

500

1000

2000

$5000 \quad 8000$

Fig. 12. Number of counts per second from all of the 1793 sources according to the pointing direction in the sky in Galactic coordinates (longitude increasing from right to left). White pixels correspond to regions where no position has been drawn. The four maps correspond to the four energy band. Inside the $\mathrm{B} 1$ law, the rate added from the sources remains

with exposure $20.48 \mathrm{~s}$ ) are summed up to build all the timescales $\left(20.48 \times 2^{n-1} \mathrm{~s}\right.$ with $\left.n=1 . .7\right)$ to reach a maximum timescale of $\sim 20 \mathrm{~min}(1310.72 \mathrm{~s})$, which is the same procedure as will be performed onboard by the image trigger.

Firstly, we do not clean the shadowgram for any noise including the CXB. Ideally for a perfectly cleaned shadowgram (or the shadowgram of an absolutely empty sky region), the distribution of the $\mathrm{S} / \mathrm{N}$ should follow a normal distribution $\mathcal{N}(0,1)$ with zero mean and standard deviation equal to 1 . In such sky images, the onboard trigger algorithm can detect new sources (such as GRBs) when the S/N exceeds a threshold of 6.5 , with a low false-trigger rate (less than one per day, Schanne 2009).

In the case where the shadowgram contains uncleaned source patterns before deconvolution, the resulting sky image will contain point sources with high $\mathrm{S} / \mathrm{N}$ and additional coding noise anywhere in the reconstructed sky where it can no longer be suppressed (because it is located away from the positions of these sources). The point sources and the coding noise levels can reach the detection threshold, resulting in false alerts. Even if the source position can be ignored during the image analysis, the coding noise widens the $\mathrm{S} / \mathrm{N}$ distribution over the whole reconstructed sky, which can lead to false triggers. To avoid this, the trigger threshold would have to be raised. Therefore, if not suppressed prior to deconvolution, the coding noise will reduce the GRB detection efficiency. Also, the standard deviation of the $\mathrm{S} / \mathrm{N}$ in the reconstructed image (excluding the positions of the point sources) is a good indicator of the presence of coding noise in this sky image; its width needs to remain as close to 1 as possible.

Figure 13 shows an example of a shadowgram exposed for $20.48 \mathrm{~s}$ in the Galactic centre (first row, left) and the associated $\mathrm{S} / \mathrm{N}$ sky image (second row, left). In the shadowgram, the mask pattern drawn by the bright source Sco X-1 is clearly visible. This pattern leads to a bright point source in the sky image and 
N. Dagoneau et al.: Onboard catalogue of known X-ray sources for SVOM/ECLAIRs
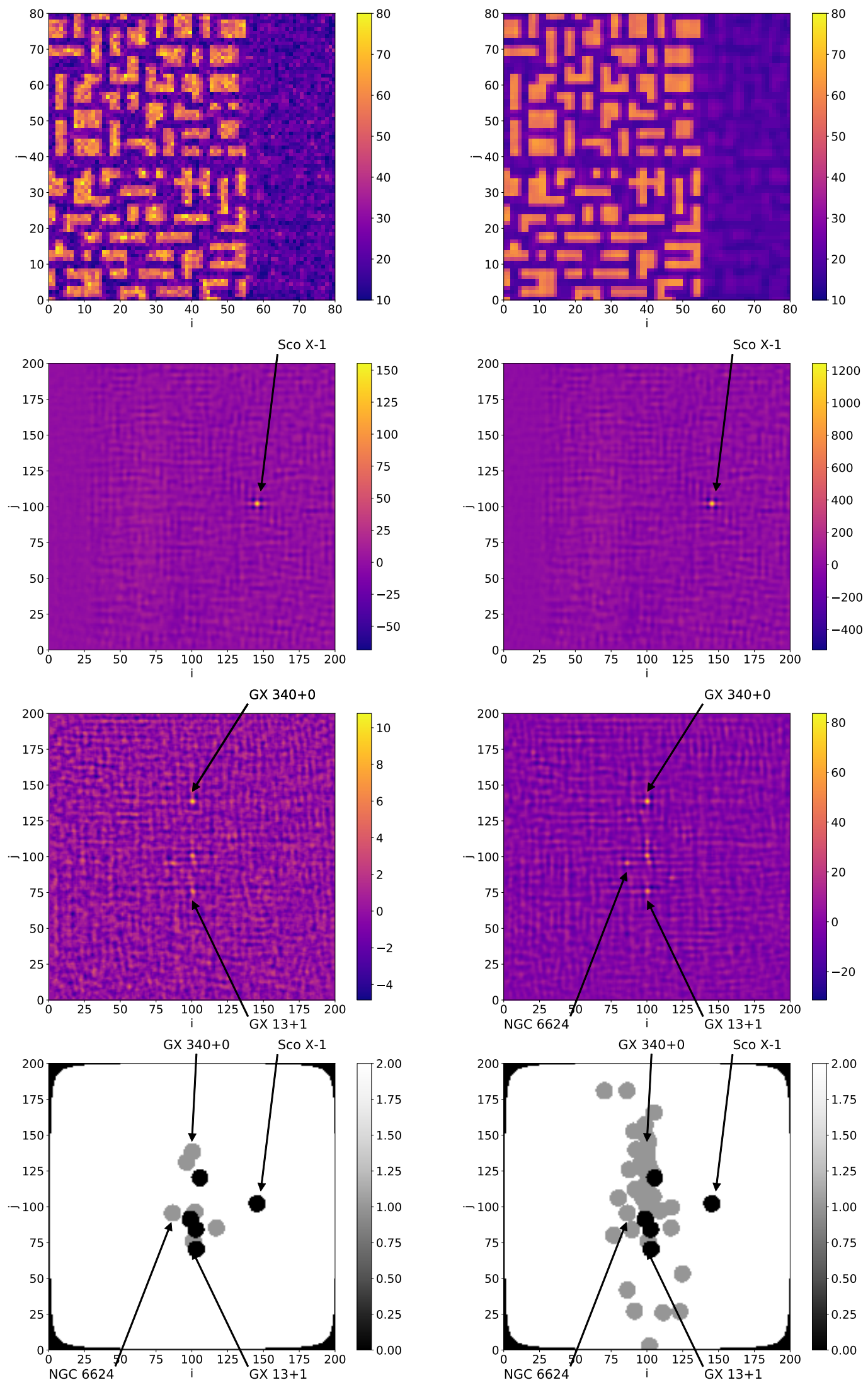

Fig. 13. First row, left: Shadowgram exposed for $20.48 \mathrm{~s}$ in the direction of the Galactic centre (4-120 keV). First row, right: corresponding weighted model used for cleaning. Second row: corresponding sky image in S/N in $20 \mathrm{~s}$ (left) and in 20 min obtained by the stacking of 64 different 20 s sky images. (right). Third row: same as above but the shadowgrams are cleaned prior to deconvolution. Last row: sources represented by black (respectively grey) circles are cleaned (respectively only masked in the sky image) in $20 \mathrm{~s}$ sky image (left) and 20 min sky image (right). 

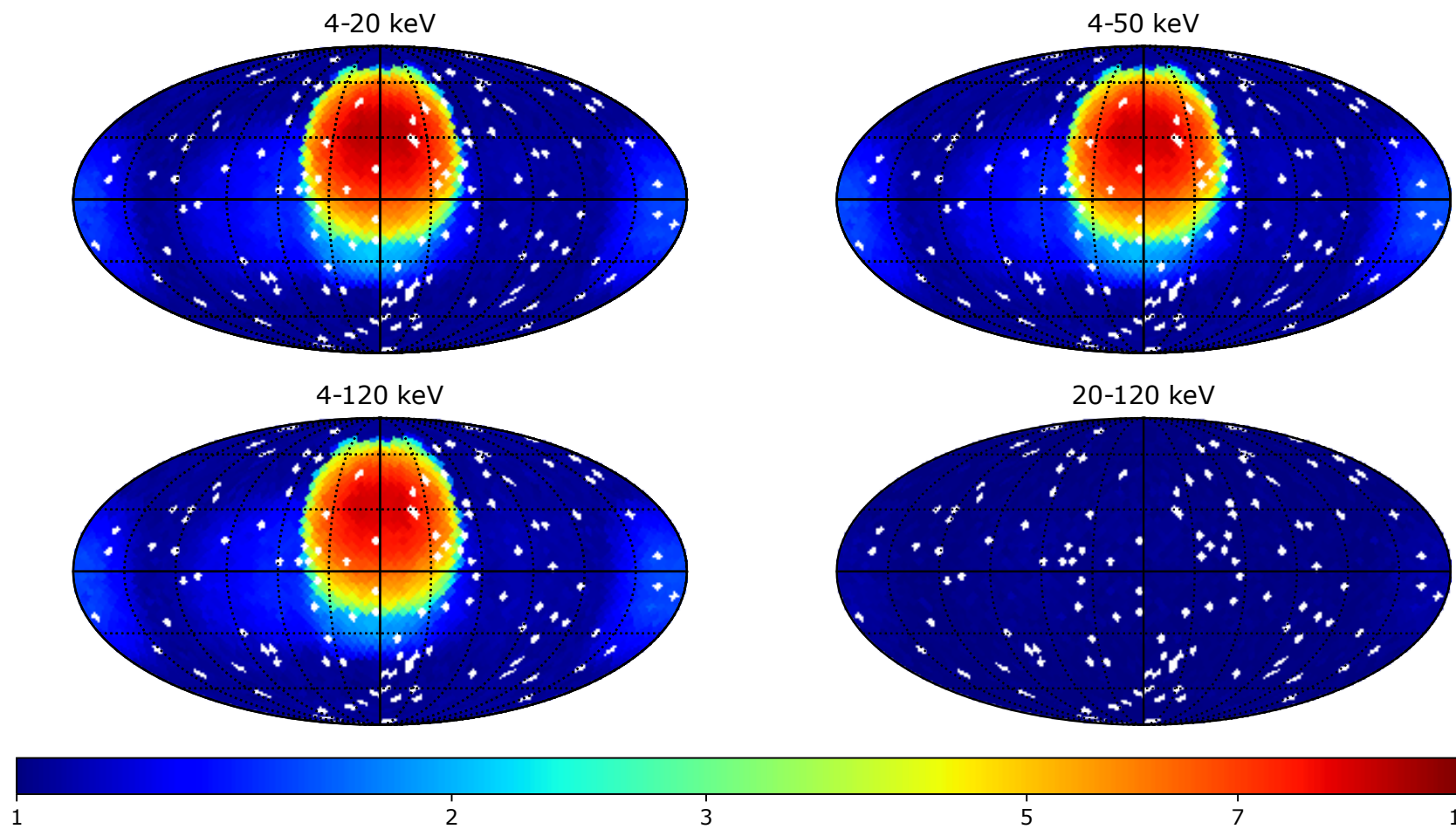

2

3

5

7

10

Fig. 14. Standard deviation of $\mathrm{S} / \mathrm{N}$ values in $20 \mathrm{~s}$ sky images according to the pointing direction in the sky in Galactic coordinates (longitude increasing from right to left). White pixels correspond to regions where no position has been drawn. The four maps correspond to the energy bands foreseen for the onboard trigger algorithm.

an $\mathrm{S} / \mathrm{N}$ distribution (outside of this point source) with a standard deviation as large as 6.06. The other bright sources in the field of view are completely drowned out by this coding noise. An image of $\sim 20 \mathrm{~min}$ is built by stacking 64 contiguous 20 s sky images and is shown in the same figure (second row, right). Its $\mathrm{S} / \mathrm{N}$ distribution outside Sco X-1 has a standard deviation of 47.45 .

Figures 14 and 15 show the S/N standard deviation according to the pointing position in Galactic coordinates for $20 \mathrm{~s}$ and 20 min sky images. In these sky images, an unsubtracted CXB background shape before deconvolution also contributes to slightly widen the $\mathrm{S} / \mathrm{N}$ distribution: in $20 \mathrm{~s}$ exposure images the unsubtracted $\mathrm{CXB}$ shape leads to a deviation of $\sim 1$, hence no cleaning of the $\mathrm{CXB}$ is required for shorter scales, while the standard deviation increases with the exposure time and reaches $\sim 2.3$ in the longest scale of $20 \mathrm{~min}(4-120 \mathrm{keV})$.

In the 20 s sky images (Fig. 14), within the B1 law the standard deviation of the $\mathrm{S} / \mathrm{N}$ is close to 1 in the four bands. Such a deviation is suitable for new source detections in sky images with weak coding noise and an $\mathrm{S} / \mathrm{N}$ threshold of 6.5. However, in the Galactic centre and near Sco X-1, the standard deviation of the $\mathrm{S} / \mathrm{N}$ reaches $\sim 10$ in the energy bands starting at $4 \mathrm{keV}$ and is no longer compatible with a detection threshold for new sources of $6.5 \%$. In the $20-120 \mathrm{keV}$ energy band, in $20 \mathrm{~s}$, the standard deviation is close to 1 for each sky pointing direction (the maximum reaches $\approx 1.1$ in the Galactic centre).

In the 20 min sky images (Fig. 15), within the B1 law the mean standard deviation of the $\mathrm{S} / \mathrm{N}$ is $\approx 2.8$ in the three first bands and $\approx 1.5$ in the $20-120 \mathrm{keV}$ band. In the Galactic centre and near Sco $\mathrm{X}-1$ the standard deviation of the $\mathrm{S} / \mathrm{N}$ reaches $\sim 70$ in the energy bands starting at $4 \mathrm{keV}$ and $\sim 2$ in the $20-120 \mathrm{keV}$ band. In any case, such a high $\mathrm{S} / \mathrm{N}$ standard deviation is not compatible with a detection threshold for new sources of $6.5 \sigma$. In these sky regions, the detection threshold would have to be raised to much higher levels.
Figure 16 shows the mean standard deviation of the $\mathrm{S} / \mathrm{N}$ for different regions of the sky: within the B1 law in dark blue or in the Galactic centre (box of $\pm 15^{\circ}$ in longitude and latitude) in red. The solid (respectively dotted) curves show the result for the simulation of the full catalogue (respectively the 53 brightest sources). In the four bands, the difference between the two simulations is very small (solid and dotted curves overlap). Within the B1 law (dark blue) the relative difference is $<2 \%$. In the Galactic centre the relative difference also remains small $(\approx 3 \%$ in the three first bands). Therefore, the contribution of the faint sources remains negligible in comparison to the bright sources. Thus, the reduced catalogue, containing the 53 brightest sources, accurately describes the influence of the sources on ECLAIRs' background level and on the standard deviation of the $\mathrm{S} / \mathrm{N}$ in the sky images. However, a simulation of the full catalogue is required to study the detectability of each source (regardless of the sensitivity).

\subsection{Source typical $S / N$}

We define the "typical" $\mathrm{S} / \mathrm{N}$ of a source to be the $\mathrm{S} / \mathrm{N}$ obtained from its simulation using the source spectrum previously determined (see Sect. 2.4) in the central pixel of the field of view of ECLAIRs with only the CXB and without any other source present. This typical $\mathrm{S} / \mathrm{N}$ is the expected upper limit of the $\mathrm{S} / \mathrm{N}$ of the sources (except in case of source outburst), because it is determined in a very favourable situation: in the part of the field of view with the best sensitivity and without the possible perturbations from other sources. The typical $\mathrm{S} / \mathrm{N}$ is given in Fig. 17 for all the sources with $S / N>6.5$ in $20 \mathrm{~min}$ and for the four energy bands foreseen. The high significance of the points comes from the fact that the sources are simulated in the centre of the field of view. An error of $15 \%$ of the $\mathrm{S} / \mathrm{N}$ is to be considered when the source position varies within the fully coded field of 

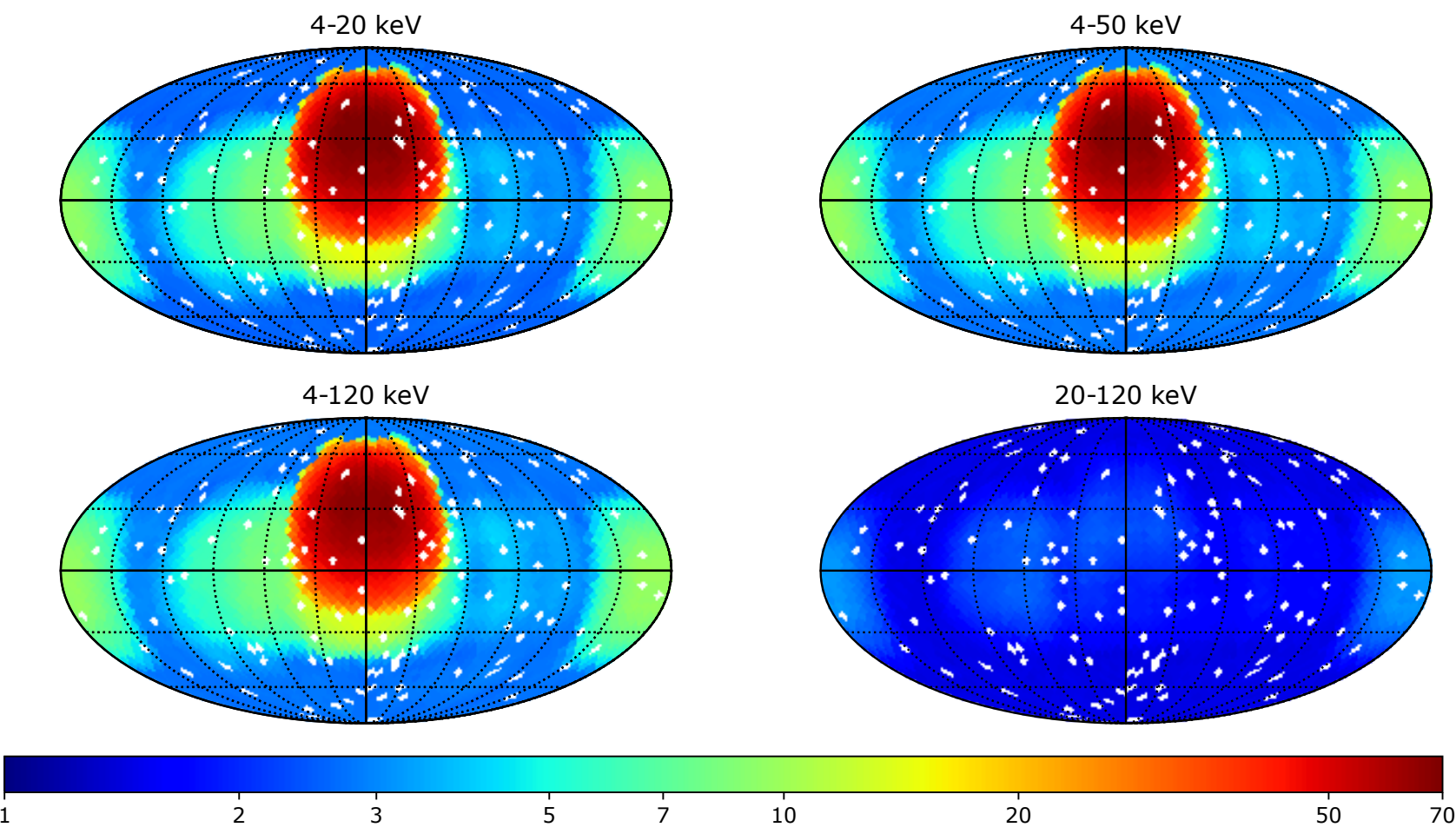

Fig. 15. Standard deviation of $\mathrm{S} / \mathrm{N}$ values in $20 \mathrm{~min}$ sky images according to the pointing direction in the sky in Galactic coordinates (longitude increasing from right to left). White pixels correspond to regions where no position has been drawn. The four maps correspond to the energy bands foreseen for the onboard trigger algorithm.
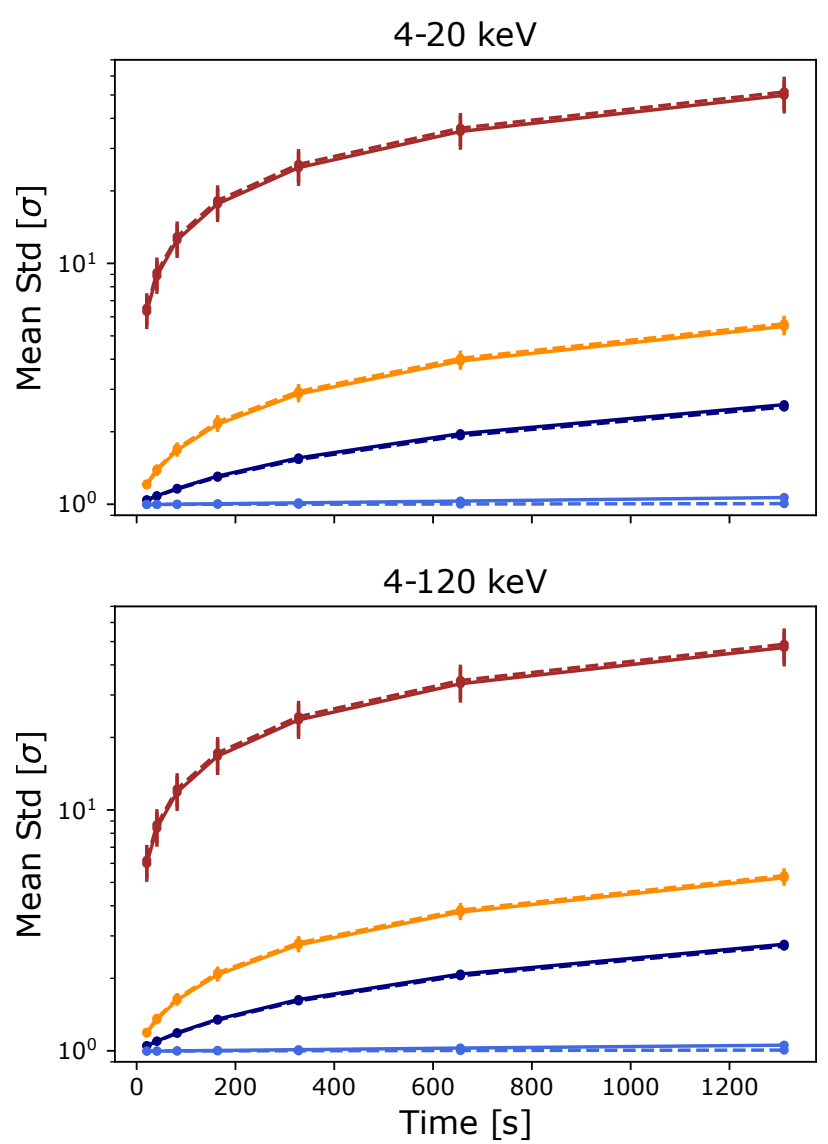

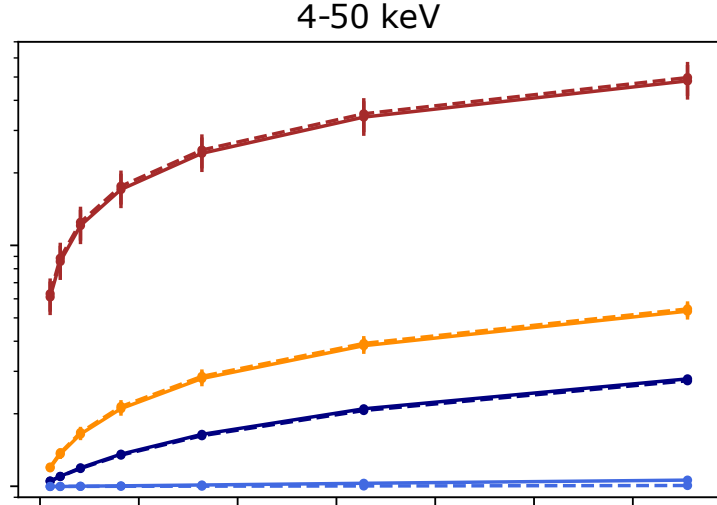

$20-120 \mathrm{keV}$

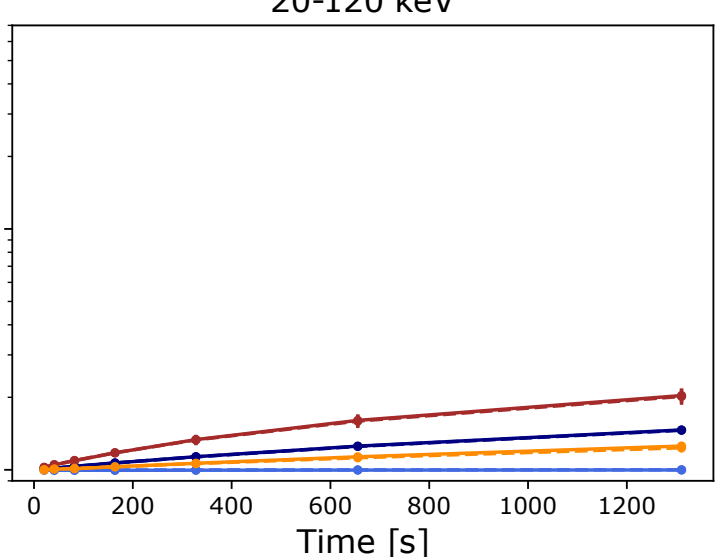

Fig. 16. Mean standard deviation of $S / N$ values in sky images from different regions in the sky: within the B1 law without cleaning (dark blue) and after cleaning (light blue, see Sect. 3.5); within the Galactic centre (box of $\pm 15^{\circ}$ in longitude and latitude) without cleaning (red), and after cleaning (orange, see Sect. 3.5). Solid lines show the simulation of the full catalogue, whereas the dotted line shows the simulation of the 53 bright sources only. The four plots correspond to energy bands foreseen for the onboard trigger algorithm. 


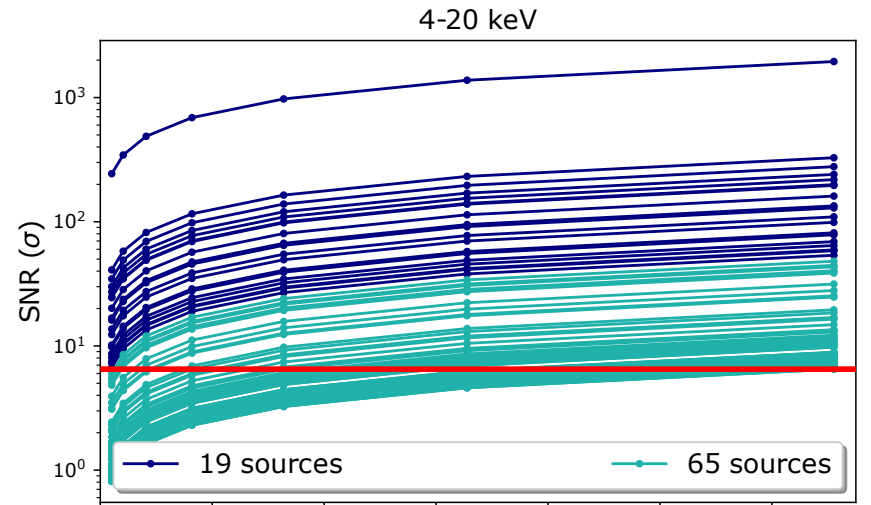

4-120 keV

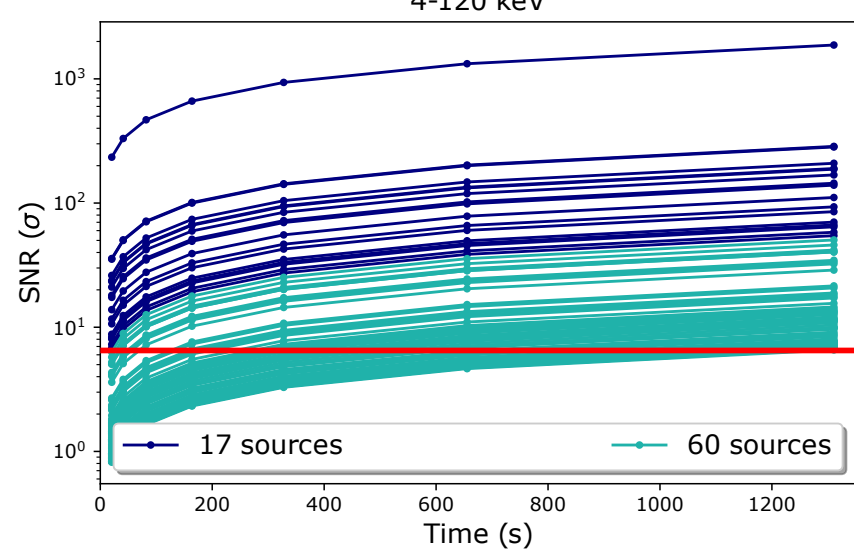

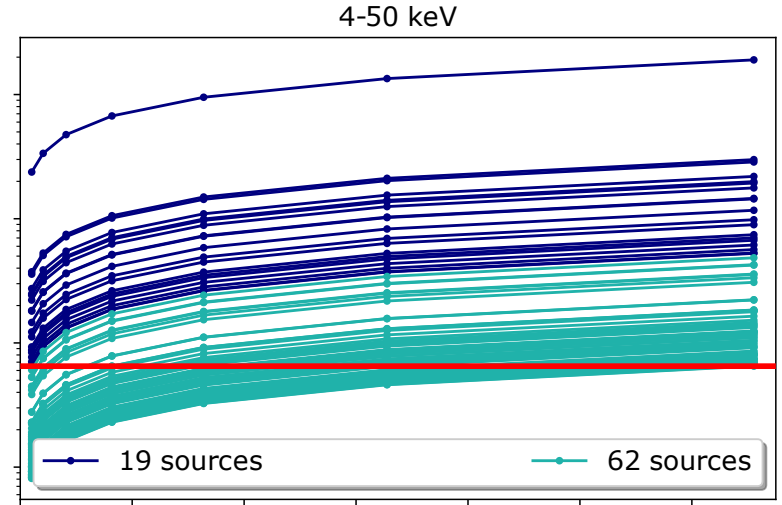

20-120 keV

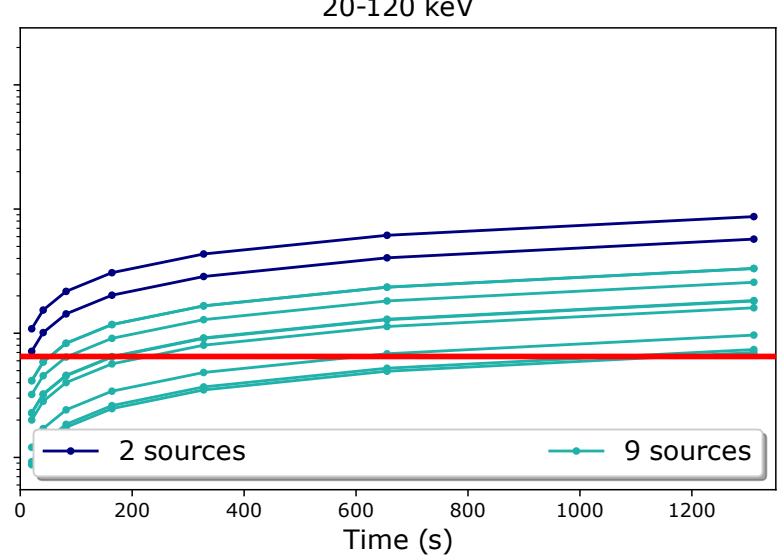

Fig. 17. Typical $\mathrm{S} / \mathrm{N}$ of the catalogue sources obtained after simulation of each source in the centre of the field of view. Dark blue (resp. cyan) curves correspond to the sources with $S / N>6.5$ in $20 \mathrm{~s}$ (resp. $20 \mathrm{~min}$ ). The red line corresponds to the threshold of $6.5 \sigma$. The four plots correspond to the energy bands foreseen for the onboard trigger algorithm.

view for all energy bands and timescales. This dispersion results from the difference of sensitivity within a sky pixel (the best sensitivity is achieved when the source is in the centre of a sky pixel).

In the four bands, there are respectively $84,81,77$, and 11 sources with a typical $\mathrm{S} / \mathrm{N}$ larger than 6.5 in $20 \mathrm{~min}$. In total, 89 sources have a typical $\mathrm{S} / \mathrm{N}$ larger than 6.5 in $20 \mathrm{~min}$ in at least one of the four bands. For these sources, the typical S/N is given in Table A.1. The number of sources in this table is larger than the estimated number of bright sources according to the sensitivity (53). Indeed, the sensitivity was determined for a specific spectrum (simple power law of index -2) with a fit of many points in the fluence-duration plane (see Fig. 11). Also, to compute the sensitivity, the dummy sources were not necessarily simulated from the centre of a pixel of the fully coded field of view. Therefore, it is possible that a source (potentially with a different spectrum) produces an $\mathrm{S} / \mathrm{N}$ larger than 6.5 even if its flux is slightly below the sensitivity limit.

\subsection{Source management and cleaning}

As we showed in the previous sections, the known sources will have an important impact on the background level, the sky image quality, and therefore the capability to detect new sources above a threshold of $6.5 \sigma$ with a low false trigger rate (Schanne 2009). An onboard cleaning step is therefore required during the imaging process. It is beyond the scope of this paper to precisely describe the methods to clean the source contributions and their performances. However, we provide in this section an example of what can be expected after cleaning. In order to demonstrate that the quality of the reconstructed sky images benefits from the cleaning of the sources, we carry out the same simulations as in Sect. 3.3 to which we add a cleaning step before the deconvolution. The shadowgrams are cleaned by fitting and subtracting a model composed of a quadratic-shape model of the CXB plus the illumination functions of up to five sources present in the field of view. The number of sources that will be modelled is limited to five because of limited computation resources onboard, and to reduce the risk of divergence of the fit. For the sources to be modelled, after each repointing, the onboard algorithm selects the five strongest sources in the field of view and computes their illumination function (projected shadowgram model) used in the fit. By "strongest sources" we mean the sources that project the largest number of photons taking into account the source flux but also the source position in the field of view (a bright source in a corner of the field of view may project less photons than a fainter source in the centre of the field of view).

Figure 13 (first row, right) shows an example of a weighted shadowgram model obtained after the fit of the raw shadowgram. The weighted model is then subtracted from the raw shadowgram to get a cleaned shadowgram ready for deconvolution. Figure 13 (third row, left) gives the sky image in S/N after deconvolution of the cleaned shadowgram. This image is to be compared with the one shown in the same figure (second row, left) where no cleaning has been performed prior to the deconvolution. The standard deviation of the $\mathrm{S} / \mathrm{N}$ in the sky region excluding the point sources is 1.14 (compared to 6.06 in the sky image 

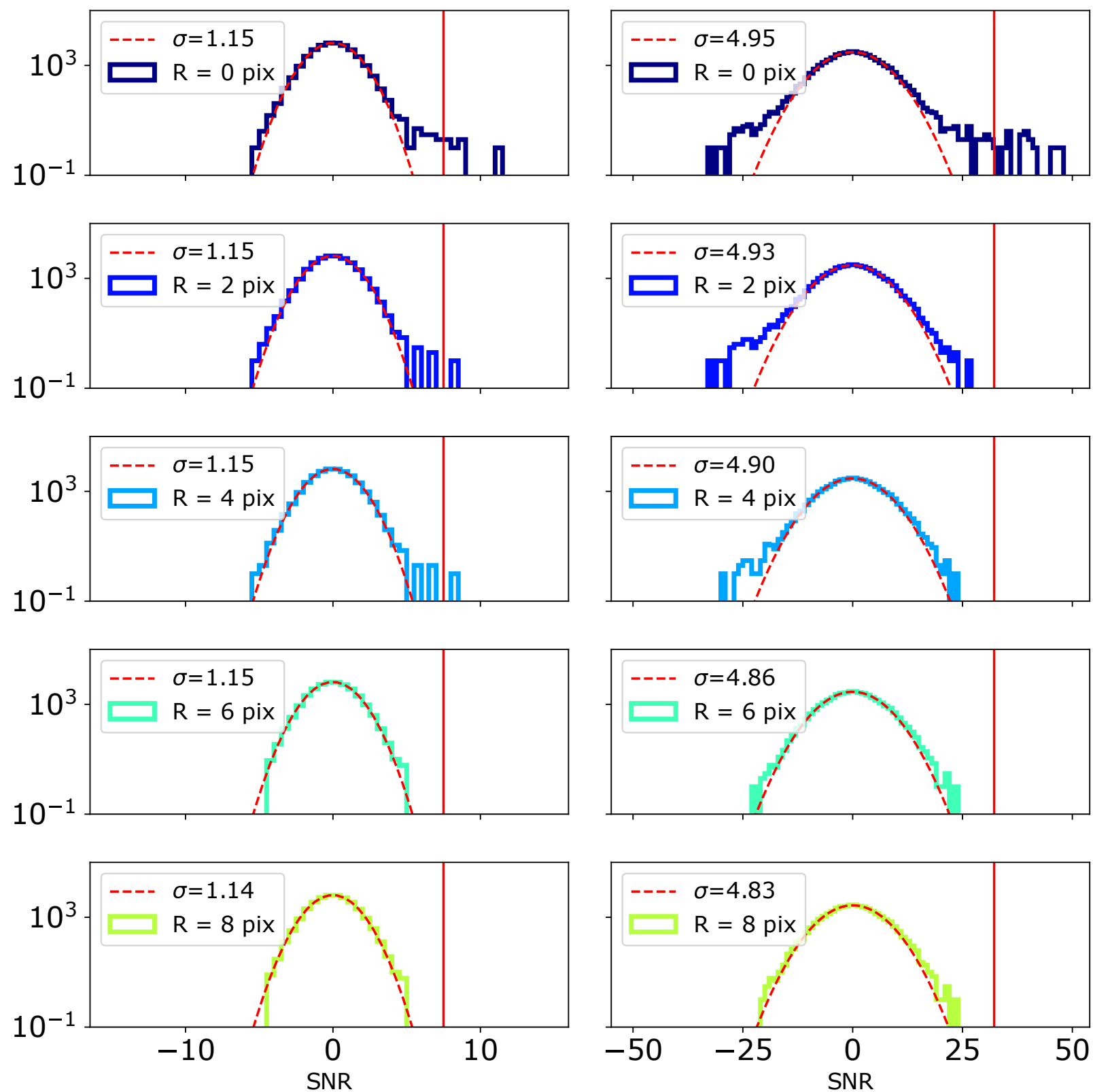

Fig. 18. Distributions of the $\mathrm{S} / \mathrm{N}$ in a sky image after deconvolution of a cleaned shadowgram for different radii used to exclude the sources. Left column: distribution of the $\mathrm{S} / \mathrm{N}$ in the $20 \mathrm{~s}$ sky image of Fig. 13 (centre). Right column: distribution in 20 min sky image (same Fig., right). The red dashed lines correspond to a Gaussian function fitted to the distribution. With these distributions, the threshold to be applied for source detection would be $1.15 \times 6.5=7.5$ for the $20 \mathrm{~s}$ case, and $4.86 \times 6.5=31.6$ for the $20 \mathrm{~min}$ case, as indicated by the red vertical lines.

from the raw shadowgram shown in the second row, left). In this shadowgram, the five sources that are cleaned are the lowmass XRBs Sco X-1, GX 5-1, GX 349+2, GX 9+1, and GX $17+2$ (represented by black circles in the last row of Fig. 13; the Galactic plane is vertical). After cleaning and deconvolution, some sources that were hidden by the Sco X-1 coding noise have now become visible (GX 340+0, GX13+1). The stacking up to 20 min reveals some fainter sources that are not cleaned in the shadowgram (Sgr X-4).

To get more details on the quality of the deconvolved sky image, which exhibits some point sources, and their coding noise in the region outside of the sources, we study the distribution of the S/N of the image pixels. Figure 18 shows the histograms of the sky images shown in Fig. 13 (left column for $20 \mathrm{~s}$ and right column for $20 \mathrm{~min}$ ) for different radii used to exclude the sources. If the point sources are not excluded (first row), the distribution is characterised by a Gaussian part plus tails of extreme $\mathrm{S} / \mathrm{N}$ values which correspond to the point sources. The tails can be removed by excluding enough pixels around the source positions. The optimal radius to exclude and efficiently remove the tails is 6 sky pixels, which suits both the $20 \mathrm{~s}$ and $20 \mathrm{~min}$ sky images. With smaller radii, residual high $\mathrm{S} / \mathrm{N}$ values too close to the strong sources may still cause false detections. Moreover, for a sky image resulting from a cleaned shadowgram deconvolution, when the point sources are excluded with radii sufficiently large to remove the tails, the distribution of the $\mathrm{S} / \mathrm{N}$ values in the image is well approximated by a Gaussian distribution of mean zero and standard deviation $\sigma$. For a Gaussian distribution, the measured standard deviation in the image is equal to the $\sigma$ of the Gaussian. Its benefit is therefore that we can configure the 

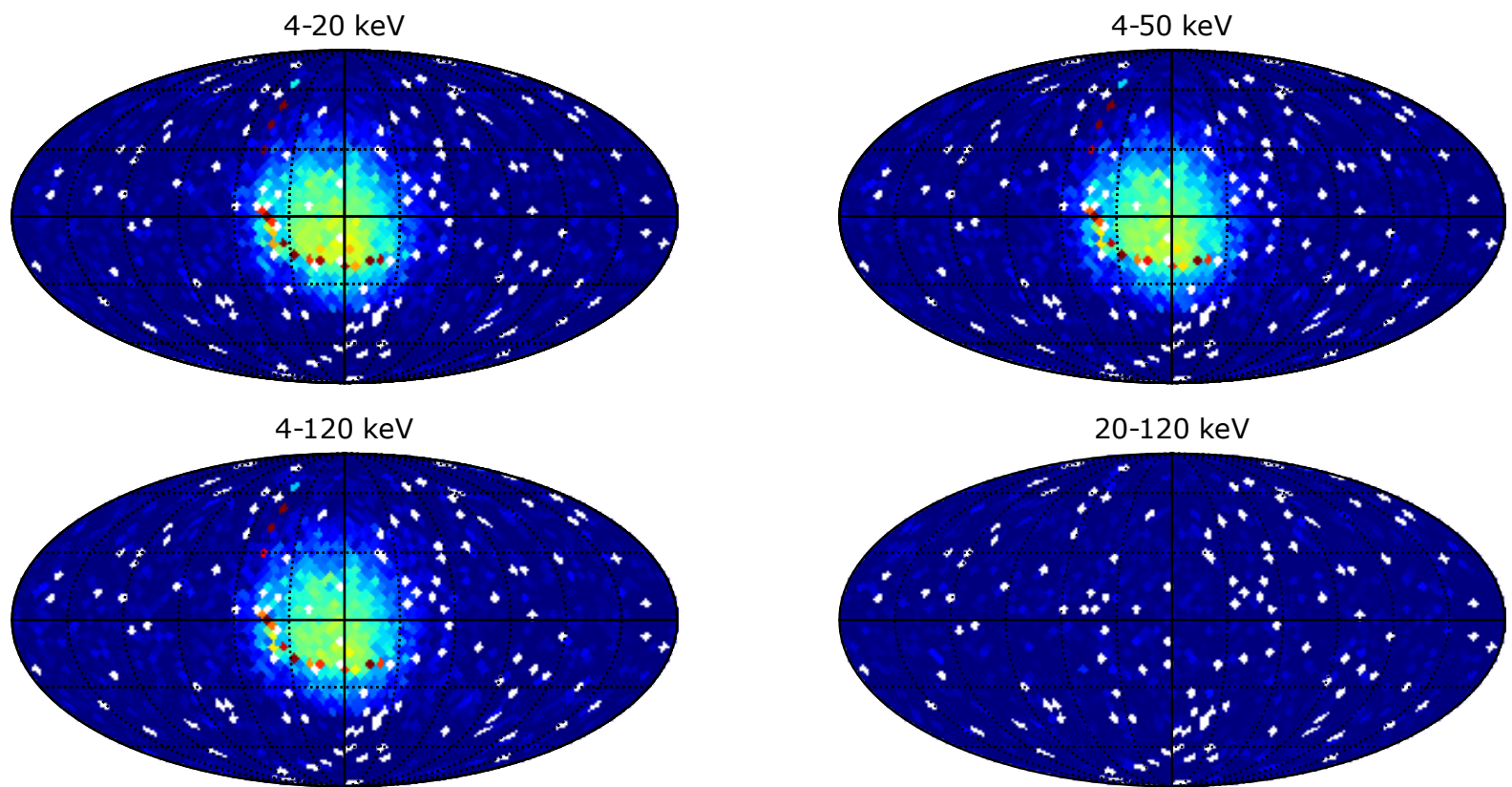

1.0

1.2

1.5

Fig. 19. Standard deviation of $S / N$ values in $20 \mathrm{~s}$ sky images (after the shadowgram cleaning) according to the pointing direction in the sky in Galactic coordinates (longitude increasing from right to left). White pixels correspond to parts where no position has been drawn. The four maps correspond to energy bands foreseen to be defined for the onboard trigger algorithm.

detection threshold as a function of this $\sigma$ to get an acceptable false alert rate.

Our analysis method will be applied on board by the trigger algorithm to be able to operate in sky regions with strong sources: after sky deconvolution, it will determine the standard deviation $\sigma$ of the distribution of the $\mathrm{S} / \mathrm{N}$ in the sky pixels excluding the sources, and adapt the trigger threshold to be above the coding noise with a safety factor (e.g. $6.5 \times \sigma)$.

Figure 19 shows the standard deviation of the S/N according to the pointing position in Galactic coordinates for $20 \mathrm{~s}$ sky images, where the shadowgrams have been cleaned prior to deconvolution the way we described previously. In the Galactic centre, in the three first energy bands, the standard deviation of the $\mathrm{S} / \mathrm{N}$ reaches $\approx 1.2$ (compared to $\approx 6$ without cleaning) whereas in the other regions it stays close to 1 . In the $20-120 \mathrm{keV}$ energy band, it remains close to 1 for all directions. The artefacts that can be seen near the Galactic centre result from the non-cleaning of Sco X-1 when this source is close to a border of the field of view and does not belong to the five sources to clean. This effect illustrates the limitations of the strategy based only on the fit to clean the sources. To prevent this issue which arises when Sco X-1 is not classified among the five strongest sources to be fitted, the detector pixels illuminated by Sco X1 can be discarded from the fit procedure and also from the deconvolution by attributing them a weight of zero. When Sco $\mathrm{X}-1$ is very partially coded, this method to suppress Sco X-1 does not significantly reduce the number of pixels active in the imaging process, and therefore does not significantly degrade the sensitivity.

Figure 20 shows the standard deviation of the $\mathrm{S} / \mathrm{N}$ according to the pointing position in Galactic coordinates for $20 \mathrm{~min}$ sky images, where the shadowgrams have been cleaned prior to deconvolution the way we described previously. Figure 16 also shows the evolution of the mean value of the standard deviation as a function of the timescale after cleaning (orange curves for the Galactic centre and light blue within the B1 law). Thanks to the cleaning, the dispersion of the $\mathrm{S} / \mathrm{N}$ for cleaned images of $20 \mathrm{~min}$ duration approaches the one obtained for images of $20 \mathrm{~s}$ without cleaning. Within the B1 law the standard deviation of the $\mathrm{S} / \mathrm{N}$ is $\approx 1.1$ in the three first bands and $\approx 1$ in the $20-120 \mathrm{keV}$ band. This is well adapted to the detection of sources above a threshold of $6.5 \sigma$. However, in the Galactic centre region, the standard deviation still reaches $\approx 5.7$ in the three first bands and $\approx 1.3$ in the last band. This sky region would require further processing if possible, or requires the use of a higher $\mathrm{S} / \mathrm{N}$ threshold to search for new sources with an acceptable false detection rate.

We would like to mention that there are possible alternatives to the method we briefly describe here. Indeed, the CXB can also be cleaned using a wavelet filtering of the shadowgrams. Moreover, a source contribution can be completely removed by attributing a weight of zero to all the detector pixels illuminated by this source in the deconvolution step (this is particularly important for Sco X-1 when it has a small coding fraction). The performances of these alternative methods will be discussed in a future paper.

\section{Onboard catalogue}

\subsection{Catalogue structure}

The details of the onboard catalogue of sources for ECLAIRs are still under development and are beyond the scope of this paper. In this section we give an overview of the possible information that will be provided in the detailed catalogue.

The catalogue table contains the sources that are bright enough to be detected by ECLAIRs in the maximum timescale of $20 \mathrm{~min}$ and deserve some processing. A flag will be given to set the processing for each source. The flag det is set for the brightest sources that can be detected in $20 \mathrm{~s}$ sky images and will be 

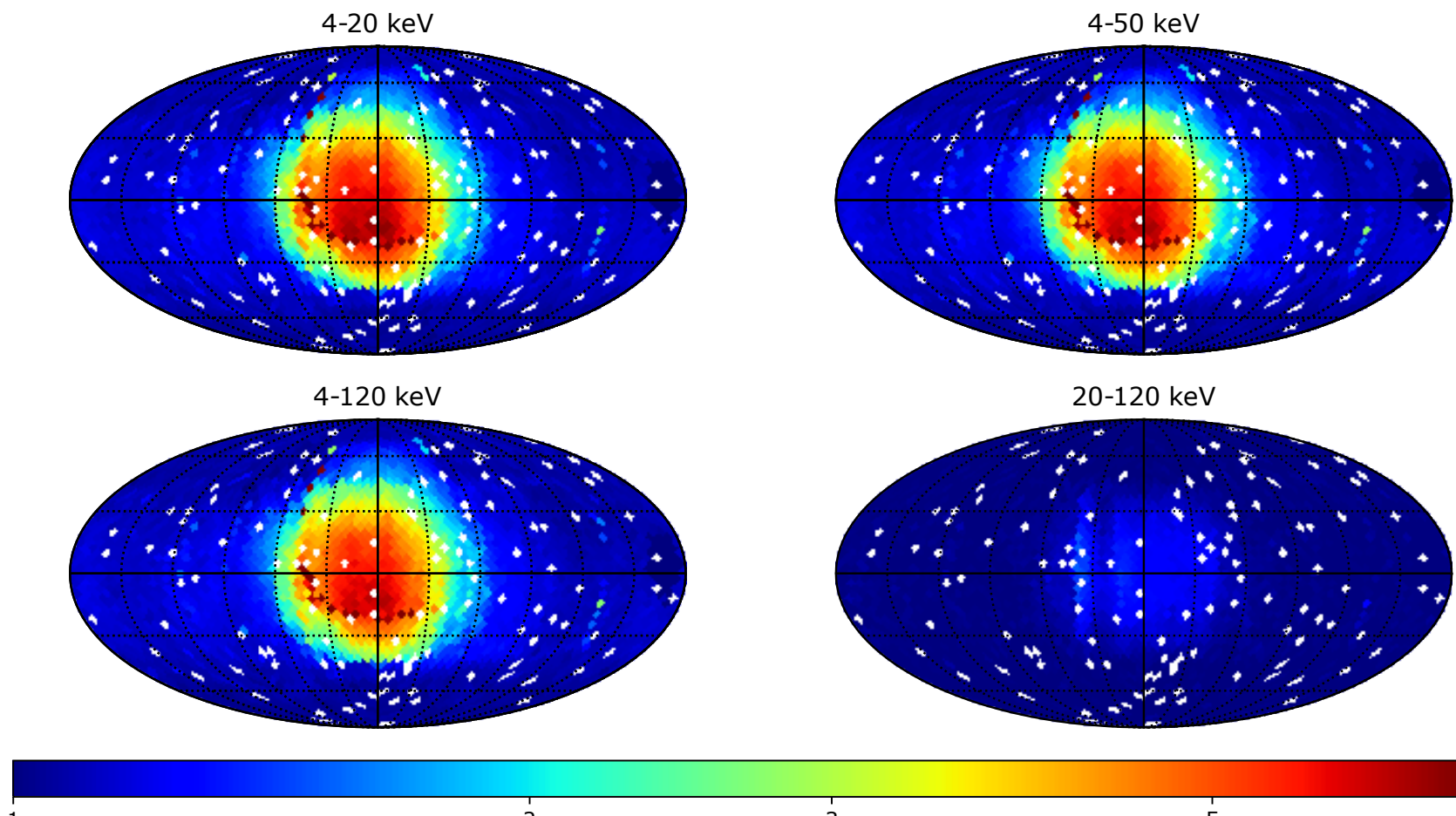

Fig. 20. Standard deviation of $\mathrm{S} / \mathrm{N}$ values in $20 \mathrm{~min}$ sky images (after the shadowgram cleaning) according to the pointing direction in the sky in Galactic coordinates (longitude increasing from right to left). White pixels correspond to parts where no position has been drawn. The four maps correspond to energy bands foreseen to be defined for the onboard trigger algorithm.

subtracted from the shadowgram using the fit method; alternatively, the detector pixels they illuminate can be discarded during the deconvolution step. The flag sky is set for the sources that can be detected in the stacked sky images up to 20 min and will be ignored in the sky image during the search for new sources. In the case where one of the sources bearing the flag $s k y$ in the catalogue is in a flaring state, the triggering system will be able to detect it above an $\mathrm{S} / \mathrm{N}$ threshold also given in the catalogue.

The flag that determines the source processing is attributed to each source according to its typical S/N (see Sect. 3.4): in $20 \mathrm{~s}$ for the det flag and in 20 min for the sky flag. Table A.1 gives the flags in the different bands for the brightest sources (the absence of a flag in one of the energy bands means that the source will not be cleaned or will be avoided in this band and thus can be detected as a new source).

Figure 21 shows the number of sources given in Table A.1 and bearing the flag det, which are present in ECLAIRs' field of view, as a function of the pointing direction in Galactic coordinates. The Galactic centre region contains most of these sources. The region of the sky in which more than five sources deserve to be fitted covers $2.68,2.62$, and $2.21 \mathrm{sr}$, respectively, in the first three energy bands (which is approximately the size of the total field of view of ECLAIRs, i.e. one-sixth of the sky). In the last band, only Crab and Cyg X-1 bear the flag det. In the case where more than five sources should be fitted, the onboard algorithm has to compute a score to determine the five best sources to be included in the fit (as discussed previously, see Sect. 3.5).

\subsection{Onboard source detection}

The $\mathrm{S} / \mathrm{N}$ threshold used to detect the outburst of a particular source (for the sources bearing the flag sky in the onboard catalogue) is based on the typical $\mathrm{S} / \mathrm{N}$ of that source (that can be multiplied by an adjustable factor $K$ ). Ideally, the threshold should be different for each source, for each energy band, and for each timescale, which leads to many parameters in the catalogue. A workaround to reduce the size of the catalogue is to set an $\mathrm{S} / \mathrm{N}$ threshold for each source, for each energy band, and for a reference timescale of $20 \mathrm{~min}$, and to compute the threshold for the other timescales onboard by applying a scaling factor $S$. The scaling factor can be the same for the four energy bands and for all the sources; it is derived from the $\mathrm{S} / \mathrm{N}$ evolution according to the exposure time (see Fig. 17), normalised by the $\mathrm{S} / \mathrm{N}$ in $20 \mathrm{~min}$. Thus, for the seven different timescales $\left(2^{n-1} \times 20.48 \mathrm{~s}\right.$ with $n=1 . .7$ ), the scaling factor $S$ is : $0.125,0.177,0.250$, $0.354,0.500,0.707,1.0$. As an example, to detect a flare from the source Ser X-1 (in 4-120 keV) whose typical S/N in $20 \mathrm{~min}$ is $\approx 50$, the $\mathrm{S} / \mathrm{N}$ threshold in an image of $20.48 \mathrm{~s}$ would be defined as $S / N_{\text {thres }}=K \times 50 \times 0.125 \times \sigma=6.25 \times K \times \sigma$. In an image from a well-cleaned shadowgram $(\sigma=1)$ and for a factor $K=1.5$ (as an example), this leads to a threshold of 9.38 (where the typical $\mathrm{S} / \mathrm{N}$ on this timescale is 6.25 ). Once a flare from this source is detected above this threshold, the typical $\mathrm{S} / \mathrm{N}$ of the source can be increased automatically onboard to the $\mathrm{S} / \mathrm{N}$ of the flare, which will provide a larger threshold for a possible future flare, and which prevents triggering multiple times on the source during its outburst which may last many hours or days.

If an event is detected and its position does not match with a source of the onboard catalogue (or there is no det or sky flag associated to the source in the triggering energy band), the observation strategy will follow the same as the one defined for GRB candidates. A faint X-ray source that is not included in the onboard catalogue may be identified on ground by a search in object databases such as SIMBAD. In this case, the detected source can be added to the onboard catalogue with a flag and a threshold (from ground by telecommand). Here, we suppose that the onboard instruments MXT and VT do not need to know whether the alert comes from such a source or from a GRB. 

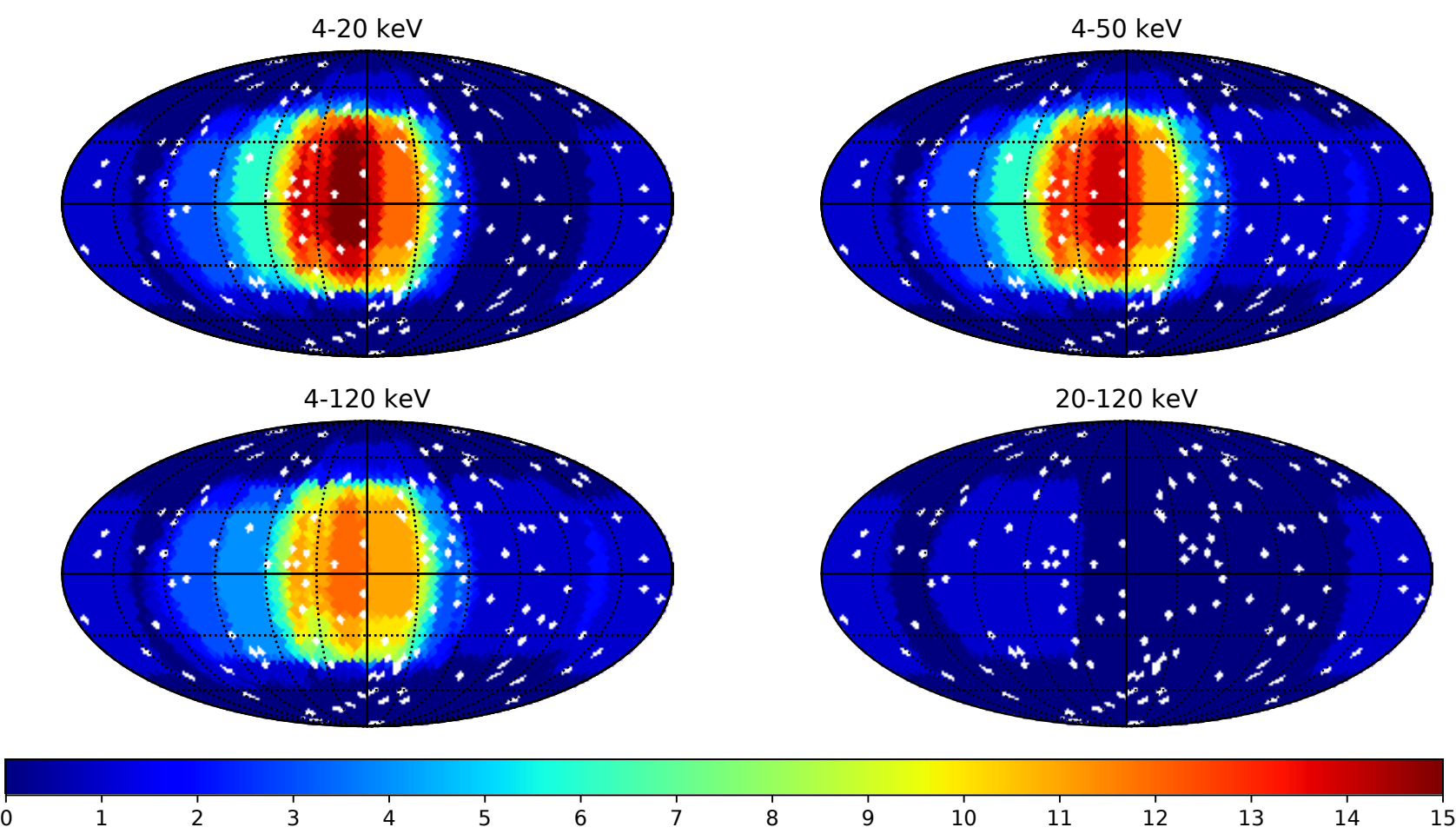

Fig. 21. Number of sources present in the ECLAIRs field of view and bearing the flag det according to the pointing direction in the sky in Galactic coordinates (longitude increasing from right to left). White pixels correspond to where no position has been drawn. The four maps correspond to the energy bands foreseen for the onboard trigger algorithm. In order to compute the number of sources per sky region, 10000 pointing positions have been drawn isotropically in the sky, with a roll angle fixed to $0^{\circ}$.

\subsection{Discussion}

The catalogue that we present from this study is a preliminary version intended for trigger studies and development. The first onboard operational version of the catalogue will depend on various parameters such as the energy bands that will be used by the triggering system, and on the strategy for the detection of outbursts that will be chosen for sources one by one.

In order to build our catalogue, we used the median flux of each source to determine a typical $\mathrm{S} / \mathrm{N}$ that allowed us to derive a processing flag and a detection threshold. However, the sources are not all steady but can exhibit different types of variability (with features such as bright outbursts or periodic flares). As a consequence, a few sources of the catalogue presented here have been marked as requiring to be cleaned or avoided only because their light curves showed a few bright flares over many years of observation (sufficiently bright to rise their median flux, leading to a typical $\mathrm{S} / \mathrm{N}$ above 6.5 in $20 \mathrm{~min}$ ). Such cases are indicated as "outbursts" in the "class of variability" attributed to each source in Table A.1, taken from Krimm et al. (2013). As an example, the source MAXI J1820+070 is an XRB that entered into a bright outburst phase between March and October, 2018 (Bharali et al. 2019). It is now in a quiet state but it may produce other outbursts in the future. These sources will be removed from the operational catalogue as they are not expected to disturb the triggering system. Some of these sources are even of particular interest for their rare flares while being otherwise very faint, and can therefore be treated onboard as unknown sources.

The flags and the thresholds for the detection of knownsource outbursts will be set in the onboard catalogue according to the values we computed, but also based on the first data that will be collected by ECLAIRs in flight. Indeed all the counts recorded by the detector will be sent to the ground, with a delay of about 6 to $12 \mathrm{~h}$, which will permit reprocessing of the data and tuning of the onboard algorithms. During the commissioning phase of the mission (in the first months after launch), it is probable that the autonomous satellite slews towards detections of outbursts of known sources will be disabled, and that, at the beginning, pointings towards the Galactic centre region will be avoided in order to focus on the tuning of the parameters for the detection of unknown sources (GRB candidates or known sources that are too faint to be in the onboard catalogue).

The onboard catalogue, its number of entries, the values it contains, its flags, and thresholds will be configurable by telecommands from the ground. In particular, once the algorithm has automatically increased the detection threshold for a known source (or included a new unknown source in the catalogue) in order to avoid repeated triggering on the same source outburst, its threshold can be reprogrammed from ground to normal values during the next routine telecommand upload, typically every week.

\section{Conclusion}

The catalogue of sources that we present in this article is composed of 1793 sources and is built from Swift/BAT and MAXI/GSC data. We used this catalogue to study the influence of the known X-ray sources on the SVOM/ECLAIRs background level and show that the source contributions will widen the distribution of the $\mathrm{S} / \mathrm{N}$ in sky images produced after deconvolution of ECLAIRs' detector images. We also produced a preliminary version of the onboard catalogue with 89 bright sources that need to be cleaned or avoided during searches for new sources.

The influence of the sources is strongly dependent on the pointing position, on the exposure time, and on the trigger energy band. This study shows that in any case, a cleaning of bright sources (such as Sco X-1) is required to reduce the coding noise 
in the sky images by as much as possible and to keep the standard deviation of the $\mathrm{S} / \mathrm{N}$ distribution in reconstructed sky images close to 1 in order to detect new sources (such as GRBs) as part of the SVOM core program with a low false trigger rate.

Acknowledgements. ECLAIRs is a cooperation between CNES, CEA and CNRS, with CNES acting as prime contractor. This work is supported by CEA and by the "IDI 2017" project of the French "Investissements d'Avenir" program, financed by IDEX Paris-Saclay, ANR-11-IDEX-0003-02. This research has made use of the SIMBAD database, operated at CDS, Strasbourg, France and of the MAXI data provided by RIKEN, JAXA and the MAXI team. We would like to acknowledge helpful comments on the manuscript from the anonymous referee. We would also like to thank very much David Palmer from the Swift/BAT onboard trigger software team for his very valuable comments and advises.

\section{References}

Baumgartner, W. H., Tueller, J., Markwardt, C. B., et al. 2013, ApJS, 207, 19 Bekhti, N. B., Flöer, L., Keller, R., et al. 2016, A\&A, 594, A116

Bharali, P., Chauhan, J., \& Boruah, K. 2019, MNRAS, 487, 5946

Connaughton, V., Briggs, M. S., Goldstein, A., et al. 2015, ApJS, 216, 32

Dagoneau, N., Schanne, S., Atteia, J.-L., Götz, D., \& Cordier, B. 2020, Exp. Astron., 50, 91

dal Fiume, D., Orlandini, M., Cusumano, G., et al. 1998, A\&A, 329, L41

Gaia Collaboration 2018, VizieR Online Data Catalog: II/345
Galloway, D. K., in 't Zand, J., Chenevez, J., et al. 2020, ApJS, 249, 32

Godet, O., Nasser, G., Atteia, J., et al. 2014, in SPIE Astronomical Telescopes + Instrumentation (Montréal, Quebec, Canada)

Goldwurm, A., David, P., Foschini, L., et al. 2003, A\&A, 411, L223

Jaubert, J., Morand, V., \& Jouret, M. 2017, Realistic Mission Scenariosfor Satellite Power Analysis, Tech. rep.

Krimm, H. A., Holland, S. T., Corbet, R. H. D., et al. 2013, ApJS, 209, 6

Le Provost, H., Schanne, S., Flouzat, C., et al. 2013, 2013 IEEE Nuclear Science Symposium and Medical Imaging Conference

Mate, S., Bouchet, L., Atteia, J.-L., et al. 2019, Exp. Astron., 48, 171

Matsuoka, M., Kawasaki, K., Ueno, S., et al. 2009, PASJ, 61, 999

Moretti, A., Pagani, C., Cusumano, G., et al. 2009, A\&A, 493, 501

Oh, K., Koss, M., Markwardt, C. B., et al. 2018, ApJS, 235, 4

Olausen, S. A., \& Kaspi, V. M. 2014, ApJS, 212, 6

Schanne, S. 2009, Presentation on the ECLAIRs Science Meeting (IAP, 2009/01/09)

Schanne, S., Le Provost, H., Kestener, P., et al. 2013, 2013 IEEE Nuclear Science Symposium and Medical Imaging Conference

Schanne, S., Cordier, B., Atteia, J. L., et al. 2015, Proceedings of Swift: 10 Years of Discovery - PoS (SWIFT 10) (SISSA Medialab), 233

Schanne, S., Dagoneau, N., Château, F., et al. 2019, Mem. Soc. Astron. It., 90, 267

Sizun, P. 2011, Synthesis of ECLAIRs Geant4 Simulations, Tech. rep.

Toor, A., \& Seward, F. D. 1974, AJ, 79, 995

Wei, J., Cordier, B., Antier, S., et al. 2016, ArXiv e-prints [arXiv: 1610.06892]

Weisskopf, M. C., Guainazzi, M., Jahoda, K., et al. 2010, ApJ, 713, 912

Wenger, M., Ochsenbein, F., Egret, D., et al. 2000, A\&AS, 143, 9

Wilson-Hodge, C. A., Case, G. L., Cherry, M. L., et al. 2012, ApJS, 201, 33

Zhao, D., Cordier, B., Sizun, P., et al. 2012, Exp. Astron., 34, 705 
A\&A 645, A18 (2021)

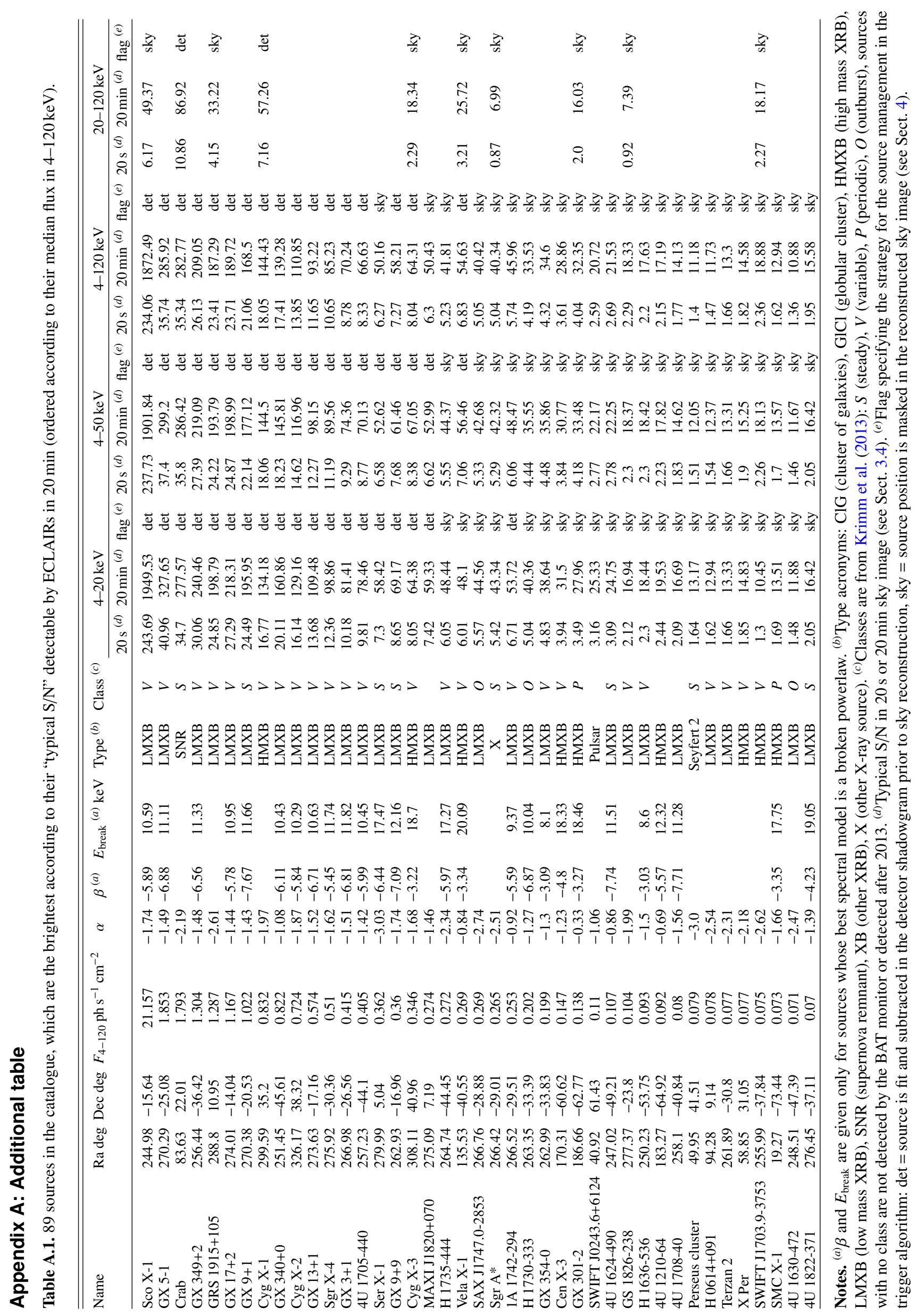


N. Dagoneau et al.: Onboard catalogue of known X-ray sources for SVOM/ECLAIRs

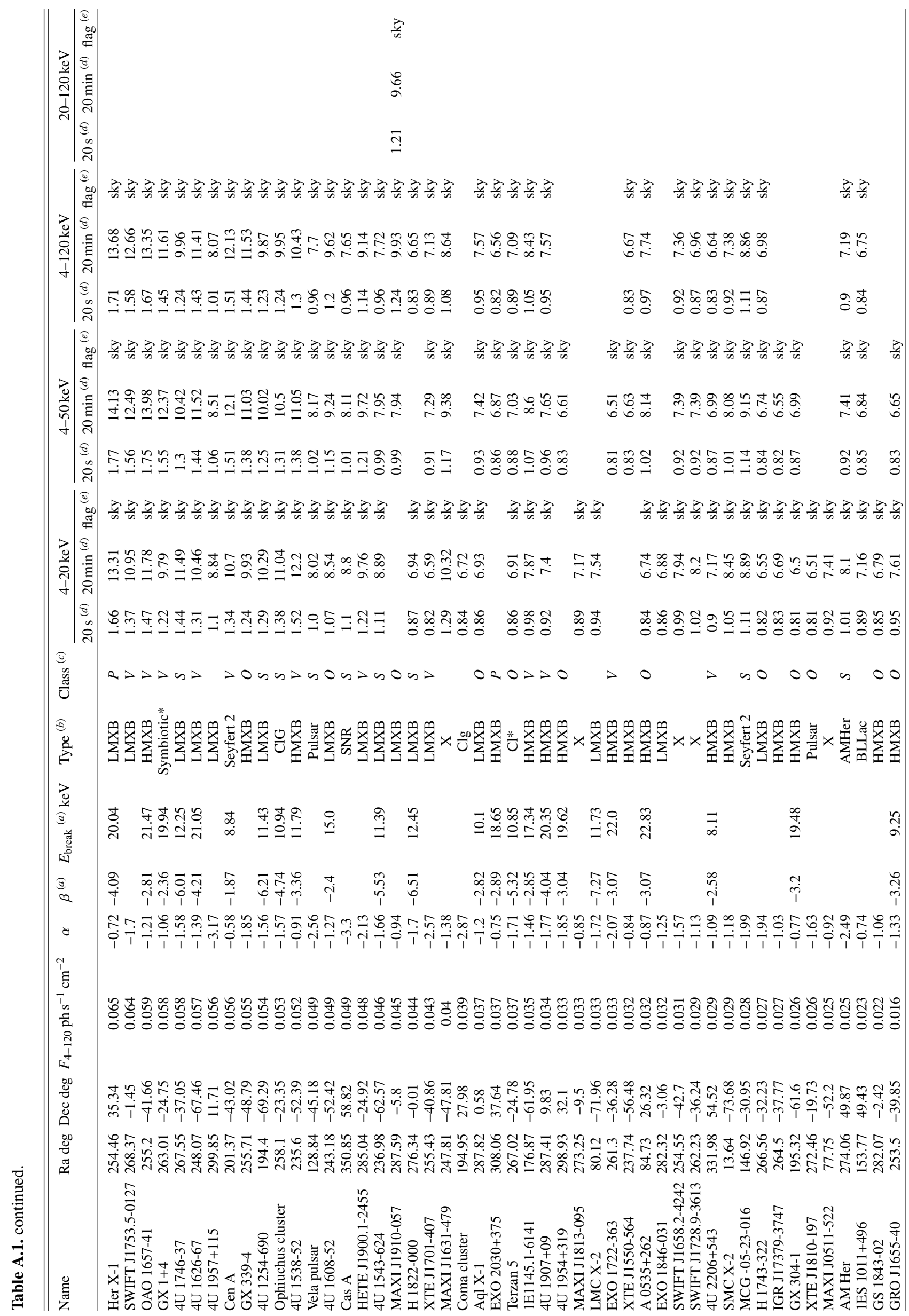

